\title{
Fractional Order Memristor No Equilibrium Chaotic System with Its Adaptive Sliding Mode Synchronization and Genetically Optimized Fractional Order PID Synchronization
}

\author{
Karthikeyan Rajagopal, Laarem Guessas, Anitha Karthikeyan, \\ Ashokkumar Srinivasan, and Girma Adam \\ Centre for Non-Linear Dynamics, Defense University, Addis Ababa, Ethiopia \\ Correspondence should be addressed to Karthikeyan Rajagopal; rkarthiekeyan@gmail.com
}

Received 12 October 2016; Revised 21 November 2016; Accepted 22 December 2016; Published 26 March 2017

Academic Editor: Ahmed G. Radwan

Copyright ( 2017 Karthikeyan Rajagopal et al. This is an open access article distributed under the Creative Commons Attribution License, which permits unrestricted use, distribution, and reproduction in any medium, provided the original work is properly cited.

This paper introduces a fractional order memristor no equilibrium (FOMNE) chaotic system and investigates its adaptive sliding mode synchronization. Firstly the dynamic properties of the integer order memristor no equilibrium system are analyzed. The fractional order memristor no equilibrium system is then derived from the integer order model. Lyapunov exponents and bifurcation with fractional order are investigated. An adaptive sliding mode control algorithm is derived to globally synchronize the identical fractional order memristor systems and genetically optimized fractional order PID controllers are designed and used to synchronize the FOMNE systems. Finally the fractional order memristor no equilibrium system is realized using FPGA.

\section{Introduction}

Chaotic systems are a special case of nonlinear systems which can be categorized as chaotic if the system possesses at least one positive Lyapunov exponent and hyperchaotic if the system possesses two or more positive Lyapunov exponents. Lyapunov exponents and fractal dimension studies are important in defining the complexity of chaotic and hyperchaotic systems [1,2]. Chaotic systems found significant importance after the discovery of a 3D weather model by Lorenz [3].

By early 21st century many researchers have announced different chaotic systems such as Chen system [4] Liu system [5], Sundarapandian system [6], Sundarapandian system [7], and Pham system [8]. Chaotic systems with no equilibrium are of great interest in chaos literature. If sum of all the Lyapunov exponents is zero then the system is a conservative system [9].

Memristors popularly known as the fourth fundamental circuit element were characterized as a nonlinear and low power device was proposed by Chua [10, 11]. In 2008
Hewlett-Packard [HP] engineers announced the first physical realization of memristors. In memristor literatures several models had been presented such as linear and nonlinear ion drift model and threshold adaptive memristor model [1214]. A Murali-Lakshmanan-Chua's circuit with a piecewise linear active flux controlled memristors with hyperchaotic behavior was investigated by Ishaq Ahamed and Lakshmanan [15]. A memristor based hyperchaotic complex Lu system and its adaptive synchronization were studied by Wang et al. [16].

Synchronization of chaotic systems is of great importance when one chaotic oscillator drives the other. Because of high sensitivity to initial conditions two identical chaotic systems may have exponentially diverging state trajectories. Many methods have been proposed in the literature such as active control method [17, 18], adaptive control method [19, 20], extended back stepping control [21, 22], sliding mode control [23, 24], and adaptive sliding mode [25-27].

Fractional calculus [28-31] has fully emerged into a mathematical field with applications in nonlinear controls, electrical and mechanical controls, and so forth. Fraction 


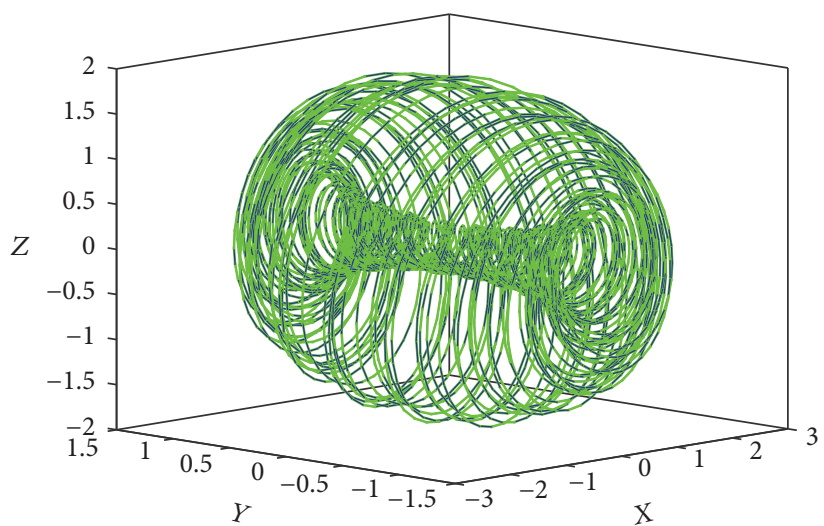

(a)

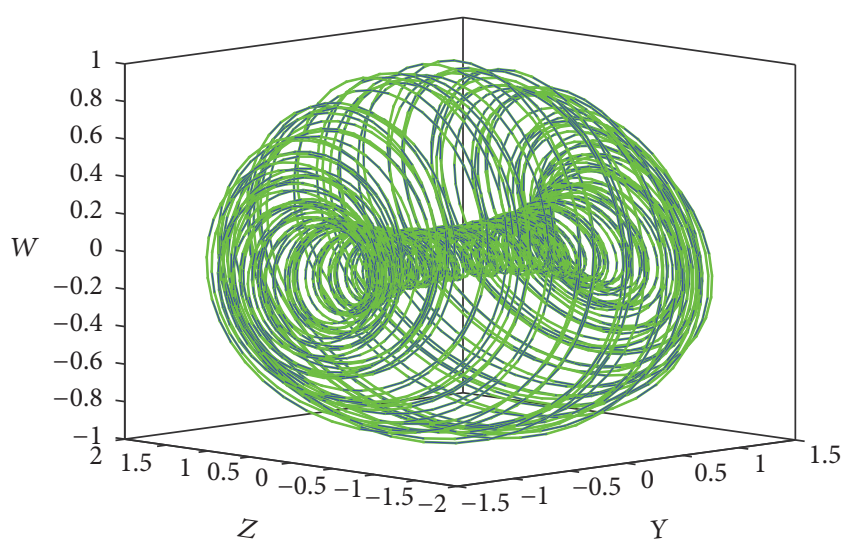

(b)

FIGURE 1: 3D state portrait of the novel memristor no equilibrium system (XYZ plane; YZW plane).

chaotic systems have been investigated by many researchers [28-35]. Fractional order controllers [24-27, 36-38] are more effective compared to its integer order models especially in chaos control and synchronization. Fractional order systems with no equilibrium are announced and investigated by $\mathrm{Li}$ and Chen [39]. For the numerical simulation of fractional order chaotic system Ivo [40] explained a methodology with a register memory component.

For chaos to exist there should be at least one unstable equilibrium point. Recently many researchers have announced chaotic systems with no equilibrium or one equilibrium [32-34]. Chaotic systems with no equilibrium exhibit hidden attractions as the orbit of attractions does not intersect with any equilibrium points [35, 41]. An algorithmic search methodology was developed by Jafari et al. [42] to discover chaotic flows with no equilibrium.

PID controllers are objects of steady effort for improvements of their quality and robustness. One of the possibilities to improve PID controllers is to use the fractional order controllers (FOPID) with noninteger derivation and integration parts; they generalize the integer order PID controller, used and verified their effectiveness in [38, 40,43-45], and add more flexibility to control design with accuracy for the real world processes. But finding appropriate parameters values for the FOPIDcontroller is still a difficult task, so in practice control engineers still often use trial and error for the tuning process.

Motivated by the above, in this paper we announce a novel integer order memristor no equilibrium chaotic system. We then derive a fractional order model of the proposed novel system. The dynamic properties of both integer order and fractional order novel systems are investigated. A fractional order adaptive sliding mode control and genetically optimized fractional order PID are proposed to synchronize the identical novel fraction order systems. Finally the proposed fractional order system is implemented in FPGA.

\section{Memristor No Equilibrium Chaotic System}

In this section we introduce a novel $4 \mathrm{D}$ memristor no equilibrium chaotic system (MNECS) with three parameters derived from the Sundarapandian system [46] by including a fourth state which is a combination of state feedback and the flux controlled memristor $[47,48]$ as described by

$$
\begin{aligned}
\dot{x} & =a z+x y \\
\dot{y} & =1-x^{2}-z^{2}-w^{2} \\
\dot{z} & =-b x+y z \\
\dot{w} & =c z-w-w(x) z
\end{aligned}
$$

and the parameter values are $a=10, b=9$, and $c=8.7$ and $w(\cdot)$ is the memductance of a flux controlled memristor characterized by the cubic nonlinearity $w(x)=a_{1}+b_{1} x^{2}, a_{1}=$ 4 , and $b_{1}=0.01$ [47].

Figure 1 shows the state portrait of system (1).

For numerical simulations, we take the initial values of the hyperchaotic system (1) as

$$
\begin{aligned}
& x(0)=1, \\
& y(0)=1, \\
& z(0)=1, \\
& w(0)=1 .
\end{aligned}
$$

\section{Properties of the 4D Memristor No Equilibrium Chaotic System}

3.1. Equilibrium Points. The equilibrium points of system (1) can be found by solving

$$
\begin{aligned}
& 0=a z+x y \\
& 0=1-x^{2}-z^{2}-w^{2} \\
& 0=-b x+y z \\
& 0=c z-w-w(x) z .
\end{aligned}
$$




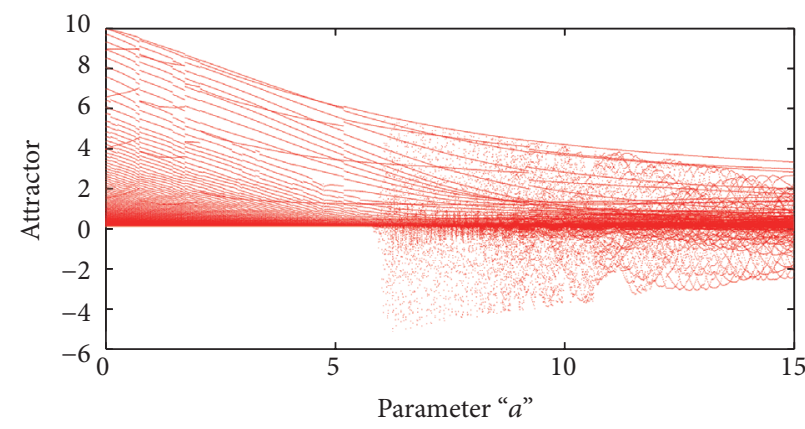

(a)

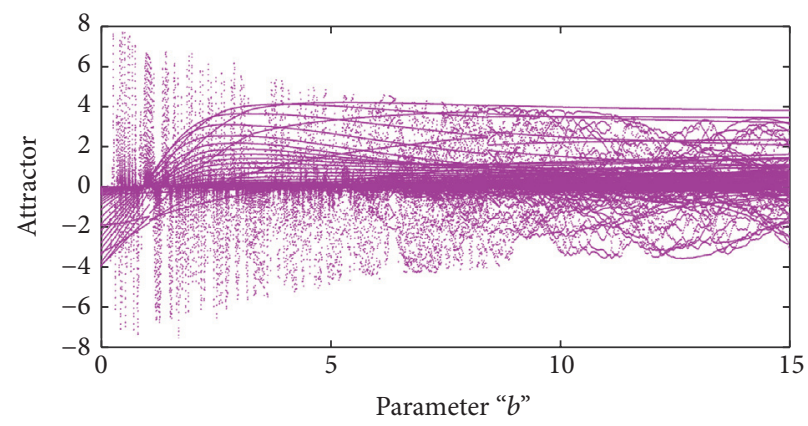

(b)

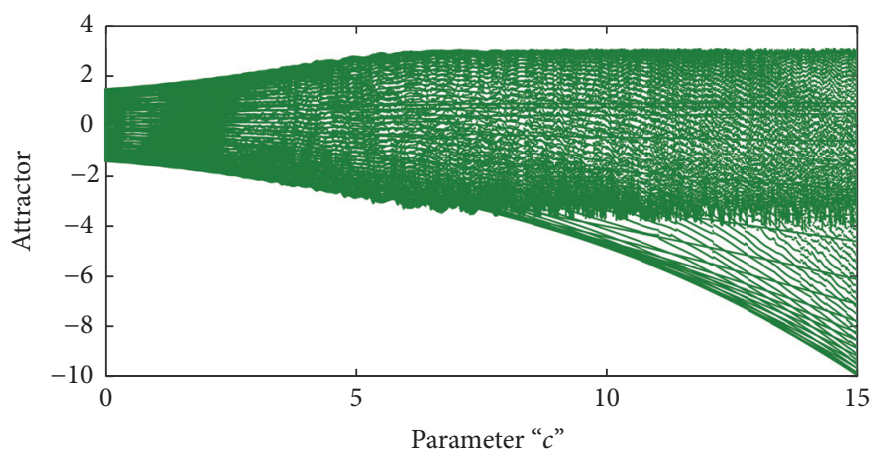

(c)

Figure 2: (a) Bifurcation plot versus $a$. (b) Bifurcation plot versus $b$. (c) Bifurcation plot versus $c$.

From (3) it can be clearly seen that $a z^{2}+b x^{2}=0$. As $a>0$ and $b>0$ the only solution is $x=z=0$. It can be seen that $c z-w-w(x) z=0$ and $x=z=0$ make $w=0$; we conclude that $y=0$. Hence system (1) has no equilibrium points.

3.2. Lyapunov Exponents and Kaplan-Yorke Dimension. The Jacobian matrix of the novel system (1) is calculated as

$$
J(X)=\left[\begin{array}{cccc}
y & x & a & 0 \\
-2 x & 0 & -2 z & -2 w \\
-b & z & y & 0 \\
0.02 x z & 0 & c-4-0.01 x^{2} & -1
\end{array}\right]
$$

The eigenvalues of the Jacobian matrix at equilibrium are $\lambda_{1}=-1.0000, \lambda_{2}=9.4868 i, \lambda_{3}=-9.4868 i$, and $\lambda_{4}=0$. The Lyapunov exponents of the system are $L_{1}=0.033426, L_{2}=0$, $L_{3}=-0.028964$, and $L_{4}=-1.005153$. The existence of positive Lyapunov exponents confirms the chaotic behavior of system (1). The sum of all the Lyapunov exponents is negative confirming that the system is dissipative.

The Kaplan-Yorke dimension of a chaotic system is defined as

$$
D_{\mathrm{KY}}=j+\sum_{i=1}^{j} \frac{L_{i}}{L_{j+1}},
$$

where $j$ is the maximum integer such that $\sum L_{i}<0$.

$$
D_{\mathrm{KY}}=3+\frac{L_{1}+L_{2}+L_{3}}{\left|L_{4}\right|}=3.004439 \text {. }
$$

3.3. Bifurcation, Bicoherence, and Poincare Map. In order to understand the dynamical behavior of the MNECS, the bifurcation plots are derived for three cases as follows.

Case 1. Fix $b=9, c=8.7, a_{1}=4$, and $b_{1}=0.01$ and vary $a$ between $\left[\begin{array}{ll}0 & 20\end{array}\right]$.

Case 2. Fix $a=10, c=8.7, a_{1}=4$, and $b_{1}=0.01$ and vary $b$ between $\left[\begin{array}{ll}0 & 20\end{array}\right]$.

Case 3. Fix $a=10, b=9, a_{1}=4$, and $b_{1}=0.01$ and vary $c$ between $\left[\begin{array}{ll}0 & 20\end{array}\right]$.

As discussed in [49], the transient behaviors occurring in memristor based nonlinear systems may result in longer simulation times to reach steady states. Hence we used the ode45 solver for numerical simulations. Figures 2(a), 2(b), and 2(c) show the bifurcation plots of the system for the parameters $a, b$, and $c$, respectively. The variation in Lyapunov exponents with reference to the parameters $a, b$, and $c$ is also derived and compared with the bifurcation plots. Figure 3 shows the variation of the Lyapunov exponents with the parameters. As it is observed from Figures 2 and 3, for the values of $8.5 \leq a, b, c \leq 10.5$, the bifurcation plots show 




FIgURe 3: Positive Lyapunov exponent for variation in parameters $(a, b, c)$.

denser points confirming the existence of a stable positive Lyapunov exponent.

The regular power spectra investigations cannot be used to analyze nonlinear systems as it does not have the required phase information. Hence researchers have adopted bicoherence or bispectrum analysis to analyze the period doubling sequence occurring in chaotic systems. Bicoherence contour of the novel system (1) as shown in Figure 4 shows the coupling between Fourier modes and is centered about the dominant frequencies composing the limit cycles [50]. As the system exhibits positive Lyapunov exponents, the chaotic behavior of the system increases resulting in period doubling and motions at additional frequencies because of the quadratic interactions between the dominant frequencies and itself. In Figure 4, the dominant frequencies are shown in a circled area which contributes to the bispectrum as indicated.

The autobispectrum with the Fourier coefficients can be derived as

$$
G\left(\omega_{1}, \omega_{2}\right)=E\left[F\left(\omega_{1}\right) F\left(\omega_{2}\right) F^{*}\left(\omega_{1}+\omega_{2}\right)\right]
$$

where $\omega_{n}$ is the radian frequency and $F$ is the Fourier coefficients of the time series. The normalized magnitude spectrum of the bispectrum known as the squared bicoherence is given by

$$
g\left(\omega_{1}, \omega_{2}\right)=\frac{\left|G\left(\omega_{1}, \omega_{2}\right)\right|^{2}}{P\left(\omega_{1}\right) P\left(\omega_{2}\right) P\left(\omega_{1}+\omega_{2}\right)},
$$

where $P\left(\omega_{1}\right)$ and $P\left(\omega_{2}\right)$ are the power spectra at $f_{1}$ and $f_{2}$.

The Poincare map is usually constructed by plotting the value of the state variable for every time it passes through a particular plane. Plotting the value every time it passes through the $x=0$ plane where $x$ is changing from negative to positive is the normal practice done when studying most

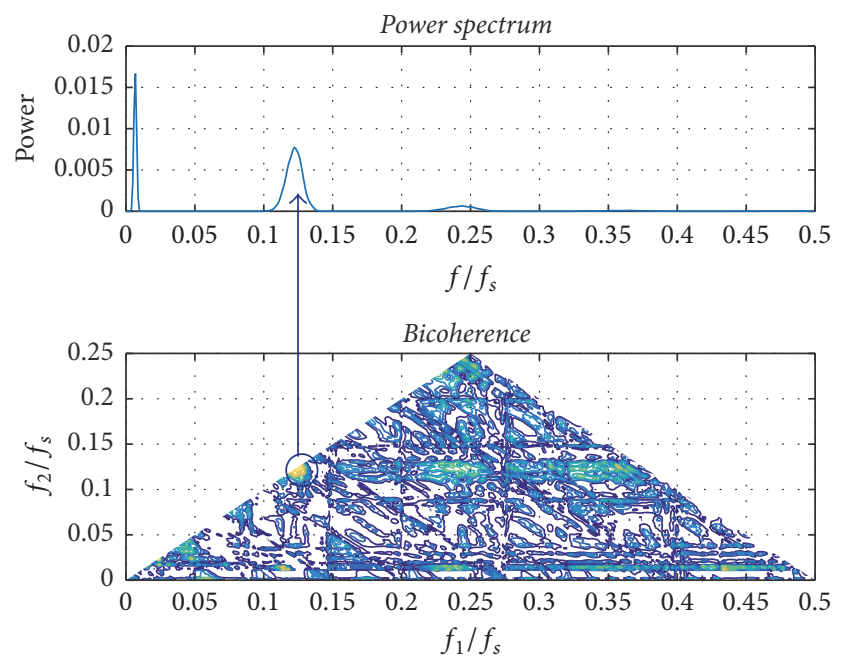

FIGURE 4: Contour of bicoherence for the novel system (1).

of the chaotic attractors. In the case of the proposed novel system (1), the regular method of plotting zero crossing of the $x$ plane is of no interest because of the nature of the system. Hence we plotted the zero crossing of system (1) with reference to the $z$ plane. Figure 5 shows the Poincare sections in the 3D state portrait of system (1). $\Sigma+$ indicates the negative to positive crossing and $\Sigma$ - indicates the positive to negative crossing in the $z$ plane.

\section{Fractional Order Memristor No Equilibrium Chaotic (FOMNE) System}

In this section we derive the fractional order model of the memristor no equilibrium system (1). There are three 




FIGURE 5: Poincare sections in the 3D state portrait of system (1) (XYZ plane).

commonly used definitions of the fractional order differential operator, Grunwald-Letnikov, Riemann-Liouville, and Caputo [28-31].

In this section, we will study the dynamical behavior of fractional order system derived from novel system (1) with the Grunwald-Letnikov (GL) definition, which is defined as

$$
\begin{aligned}
{ }_{a} D_{t}^{q} f(t) & =\lim _{h \rightarrow 0}\left\{\frac{1}{h^{q}} \sum_{j=0}^{[(t-a) / h]}(-1)^{j}\left(\begin{array}{l}
q \\
j
\end{array}\right) f(t-j h)\right\} \\
& =\lim _{h \rightarrow 0}\left\{\frac{1}{h^{\alpha}} \Delta_{h}^{q} f(t)\right\},
\end{aligned}
$$

where $a$ and $t$ are limits of the fractional order equation, $\Delta_{h}^{q} f(t)$ is generalized difference, $h$ is the step size, and $q$ is the fractional order of the differential equation. fied as

For numerical calculations the above equation is modi-

$$
{ }_{(t-L)} D_{t}^{q} f(t)=\lim _{h \rightarrow 0}\left\{h^{-q} \sum_{j=0}^{N(t)} b_{j}(f(t-j h))\right\} .
$$

Theoretically fractional order differential equations use infinite memory. Hence when we want to numerically calculate or simulate the fractional order equations we have to use finite memory principal, where $L$ is the memory length and $h$ is the time sampling.

$$
N(t)=\min \left\{\left[\frac{t}{h}\right],\left[\frac{L}{h}\right]\right\} .
$$

The binomial coefficients required for the numerical simulation are calculated as

$$
b_{j}=\left(1-\frac{a+\alpha}{j}\right) b_{j-1} .
$$

Using (10), the fractional order model of system (1) is derived as

$$
\begin{aligned}
D^{q_{x}} x & =a z+x y \\
D^{q_{y}} y & =1-x^{2}-z^{2}-w^{2} \\
D^{q_{z}} z & =-b x+y z \\
D^{q_{w}} w & =c z-w-z w(x),
\end{aligned}
$$

where $a=10, b=9, c=8.7, a_{1}=4, b_{1}=0.01, q_{x}, q_{y}, q_{z}$, and $q_{w}$ are the fractional orders of the system, and $w(\cdot)$ is the memductance of a flux controller memristor characterized by the nonlinearity $w(x)=a_{1}+b_{1} x^{2}, a_{1}=4$, and $b_{1}=0.01$ [46]. For numerical simulations, we take the initial values of the hyperchaotic system (1) as $x(0)=1, y(0)=1, z(0)=1$, and $w(0)=1$. Figure 6 shows the $3 \mathrm{D}$ state portrait of the fractional order system (13) for $q_{x}=q_{y}=q_{z}=q_{w}=0.99$.

\section{Stability Analysis of FOMNE System}

As the FOMNE system has no defined equilibrium points, direct calculation of eigenvalues is impossible and hence we consider the origin $[0,0,0,0]$ as the only time bound trivial solution and use it for stability analysis of the system.

5.1. Commensurate Order. For commensurate FOMNE system of order $q$, the system is stable and exhibits chaotic oscillations if $\left|\arg \left(\operatorname{eig}\left(J_{E}\right)\right)\right|=\left|\arg \left(\lambda_{i}\right)\right|>q \pi / 2$, where $J_{E}$ is the Jacobian matrix at the equilibrium $E$ and $\lambda_{i}$ are the eigenvalues of the FOMNE system where $i=1,2,3,4$. As seen from the FOMNE system, the eigenvalues should remain in the unstable region and the necessary condition for the 


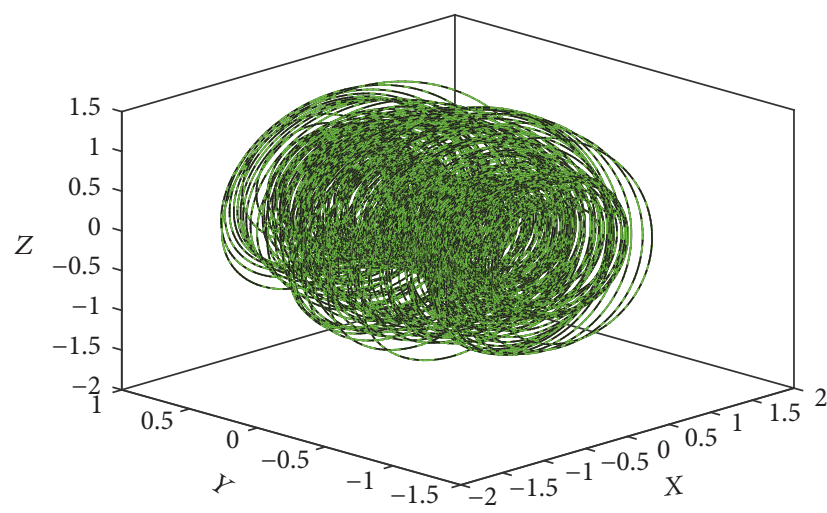

(a)

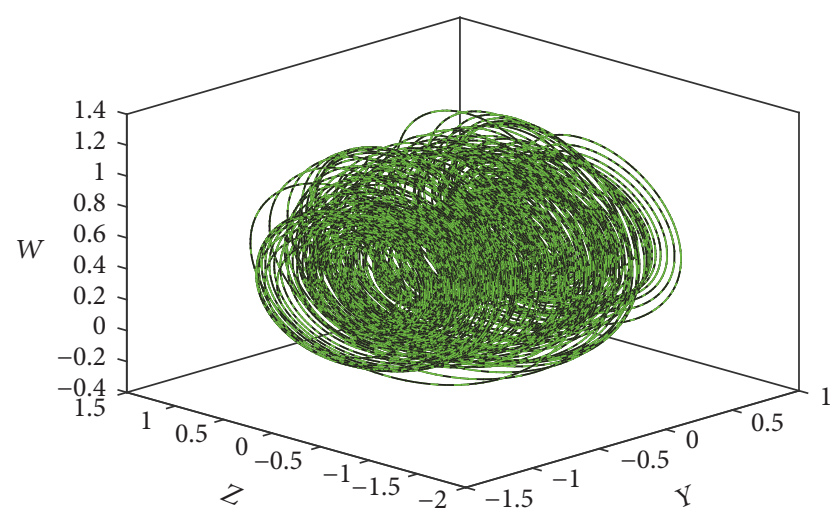

(b)

Figure 6: 3D state portrait of the FOMNE system ( $X Y Z$ plane; YZW plane).

FOMNE system to be stable is $q>(2 / \pi) \tan ^{-1}(|\operatorname{Im} \lambda| / \operatorname{Re} \lambda)$. As the eigenvalues of the system are $\lambda_{1}=-1.0000, \lambda_{2}=$ $9.4868 i, \lambda_{3}=-9.4868 i$, and $\lambda_{4}=0$, it is clearly seen that the value $\lambda_{2}$ is a saddle point and remains in the unstable region contributing to the existence of chaotic oscillations.

5.2. Incommensurate Order. The necessary condition for the FOMNE system to exhibit chaotic oscillations in the incommensurate case is $\pi / 2 M-\min _{i}\left(\left|\arg \left(\lambda_{i}\right)\right|\right)>0$, where $M$ is the LCM of the fractional orders. If $q_{x}=0.9$, $q_{y}=0.9, q_{z}=0.8$, and $q_{w}=0.8$, then $M=10$. The characteristic equation of the system evaluated at the equilibrium is $\operatorname{det}\left(\operatorname{diag}\left[\lambda^{M q_{x}}, \lambda^{M q_{y}}, \lambda^{M q_{z}}, \lambda^{M q_{w}}\right]-J_{E}\right)=0$ and by substituting the values of $M$ and the fractional orders $\operatorname{det}\left(\operatorname{diag}\left[\lambda^{9}, \lambda^{9}, \lambda^{8}, \lambda^{8}\right]-J_{E}\right)=0$, the characteristic equation is $\lambda^{34}-2 \lambda^{27}-3 \lambda^{26}+\lambda^{20}+5 \lambda^{19}+\lambda^{18}+90 \lambda^{17}-2 \lambda^{12}-4 \lambda^{11}-91 \lambda^{10}-$ $180 \lambda^{9}+\lambda^{4}+\lambda^{3}+90 \lambda^{2}+90 \lambda$. The approximated solution of the characteristic equation is $\lambda_{34}=1.414$ whose argument is zero which is the minimum argument and hence the stability necessary condition becomes $\pi / 20-0>0$ which solves for $0.0785>0$ and hence the FOMNE system is stable and chaos exists in the incommensurate system.

\section{Dynamic Analysis of the Fractional Order Memristor No Equilibrium System}

Most of the dynamic properties of the integer order system (1) like the Lyapunov exponents, eigenvalues, and bifurcation with parameters are preserved in the fractional order if $q_{i}>$ 0.90 , where $i=x, y, z, w$. The most important analysis of interest when investigating a fractional order system is the bifurcation with fractional order. The resistance of the memristor increases from the initial value until it reaches its maximum $R_{\text {off }}$ in a certain time period which is called the saturation time $t_{\text {sat }}$ [51]. The fractional order parameter can be used to control the saturation time from a part of a second up to several minutes under the same input voltage $[51,52]$. The saturation time can be controlled through the fractional order where it can be less than 1 sec when $\alpha<0.5$ up to higher values when $\alpha>0.5$. This specific character of the fractional order memristor is useful in determining the largest positive Lyapunov exponent of the system. Figure 7 shows the $3 \mathrm{D}$ state portrait ( $X Y Z$ plane) of the FOMNE system for various fractional orders $q_{x}=q_{y}=q_{z}=$ $q_{w}=q$. The largest positive Lyapunov exponent $\left(L_{1}=\right.$ 0.038174 ) of the FOMNE system appears when $q=0.99$ against its largest integer order Lyapunov exponent $\left(L_{1}=\right.$ $0.033426)$. Hence fractional order chaos suppression/control and synchronization prove efficient than the integer order controls as the systems show the largest positive Lyapunov exponent in fractional order close to 1 . It can also be seen that, as the fractional order $q$ decreases, the FOMNE system starts losing its largest positive Lyapunov exponent. When $q \leq 0.9$ the only positive Lyapunov exponent of the system becomes negative and thus the chaotic oscillations in the system disappear.

\section{Adaptive Fractional Order Sliding Mode Synchronization}

In this section we investigate the adaptive fractional order sliding mode synchronization of identical systems. Let us define a generalized fractional order master system as

$$
D^{q_{m}} x_{m}=f\left(x_{m}\right)+F\left(x_{m}\right) a,
$$

where $q_{m}$ is the fractional order of the master system and generalized slave system with adaptive controller as

$$
D^{q_{s}} x_{s}=g\left(x_{s}\right)+G\left(x_{s}\right) b+u(t),
$$

where $u(t)$ is the adaptive controller for synchronizing the nonidentical systems and $q_{s}$ is the fractional order of the slave system.

Let us define the synchronization error as

$$
e=x_{s}-x_{m}
$$




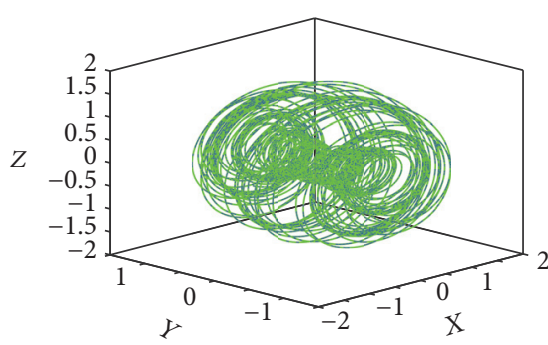

$q=0.99, L_{1}=0.033426$

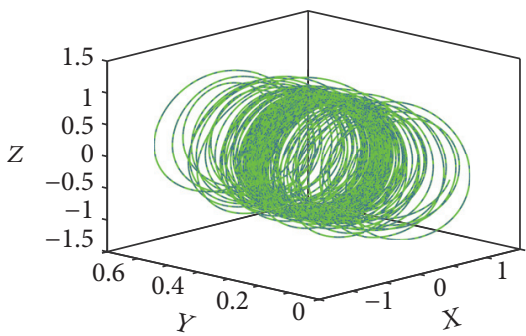

$q=0.96, L_{1}=0.030526$

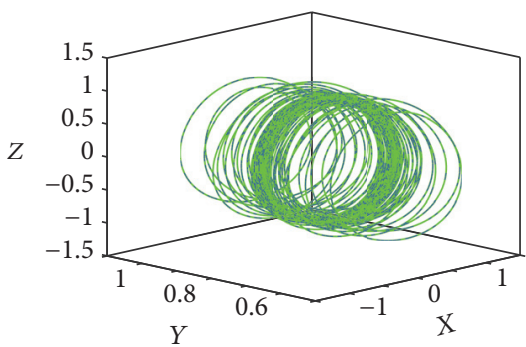

$q=0.93, L_{1}=0.022154$

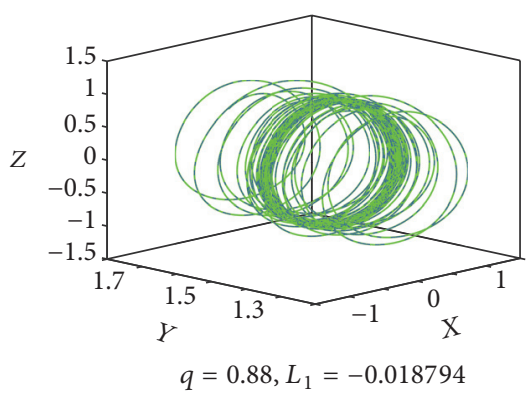

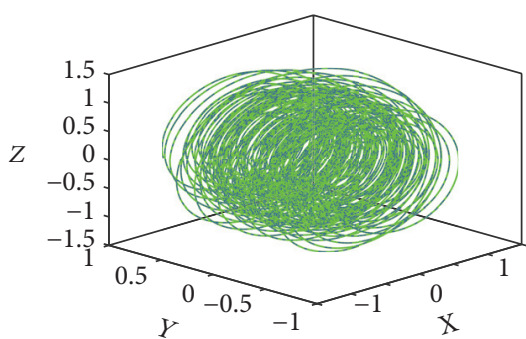

$q=0.98, L_{1}=0.032131$

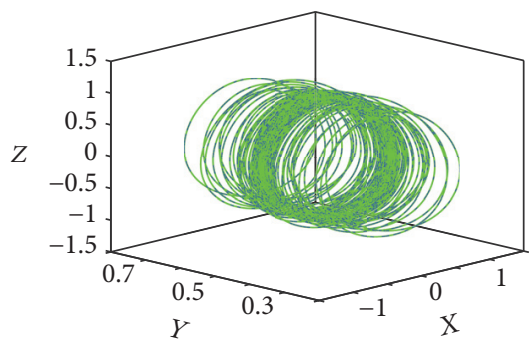

$q=0.95, L_{1}=0.029128$

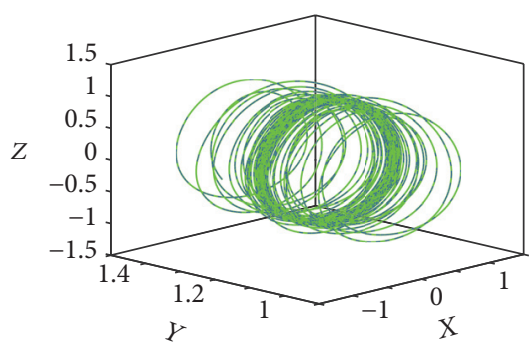

$q=0.90, L_{1}=0.019401$

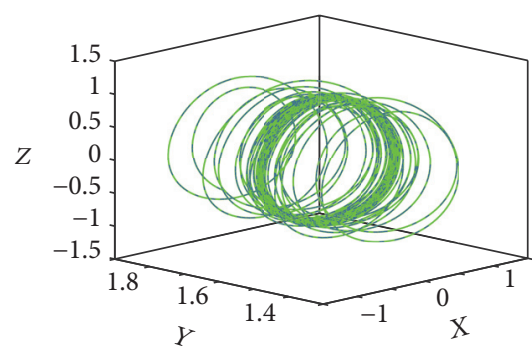

$q=0.87, L_{1}=-0.028603$

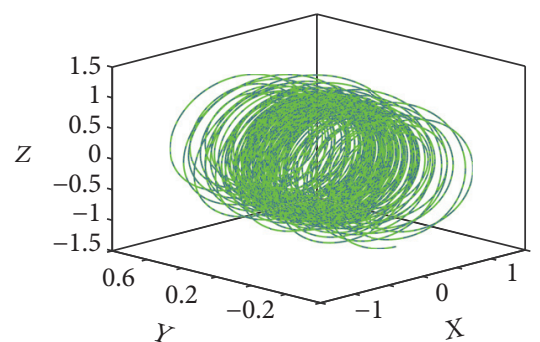

$q=0.97, L_{1}=0.031675$

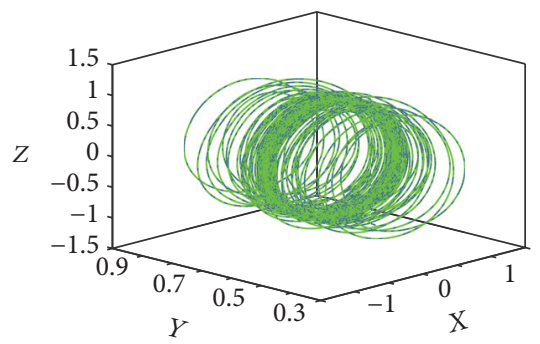

$q=0.94, L_{1}=0.026437$

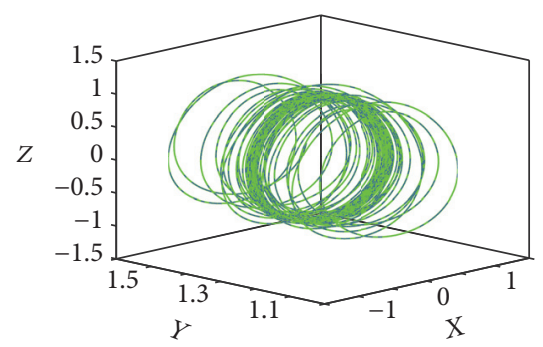

$q=0.89, L_{1}=-0.016305$

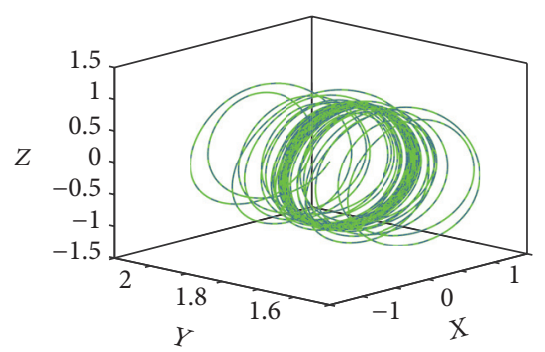

$q=0.86, L_{1}=-0.031956$

FIgURE 7: 3D state portrait ( $X Y Z$ plane) of FOMNE system for various fractional orders.

The sliding surface for the integral sliding mode control [26, $27,36]$ is defined as

$$
s(e)=e+k \int e(\tau) d \tau
$$

The fractional first derivative of the sliding surface is derived as

$$
D^{q} s=D^{q} e+k e .
$$

The fractional order error dynamics are defined as

$$
D^{q} e=D^{q_{s}} x_{s}-D^{q_{m}} x_{m} .
$$

Using (14) and (15) in (19),

$$
D^{q} e=g\left(x_{s}\right)+G\left(x_{s}\right) b+u(t)-f\left(x_{m}\right)-F\left(x_{m}\right) a .
$$

Let us define the adaptive controller as

$$
\begin{aligned}
u(t)= & -g\left(x_{s}\right)-G\left(x_{s}\right) \hat{b}+f\left(x_{m}\right)-F\left(x_{m}\right) \hat{a}-k e \\
& -\eta \operatorname{sgn}(s)-\rho s,
\end{aligned}
$$

where $k, \eta$, and $\rho$ are positive gain values, $\widehat{a}$ and $\widehat{b}$ are parameter estimates of master and slave systems, and $s$ is the sliding surface. 
Using (21) in (20), the error dynamics simplify to

$$
\begin{aligned}
D^{q} e= & G\left(x_{s}\right)[b-\hat{b}]-F\left(x_{m}\right)[a-\widehat{a}]-\eta \operatorname{sgn}(s) \\
& -\rho s .
\end{aligned}
$$

The Lyapunov candidate function to analyze the stability of the controller is defined as

$$
V=\frac{1}{2} s^{2}+\frac{1}{2}(b-\widehat{b})^{2}+\frac{1}{2}(a-\widehat{a})^{2} .
$$

The Lyapunov first derivate is derived as

$$
\dot{V}=s \cdot \dot{s}+(b-\widehat{b})(-\dot{\hat{b}})+(a-\widehat{a})(-\dot{\hat{a}}) .
$$

By definition of fractional calculus [29, 30],

$$
\dot{x}(t)=D_{t}^{1-q} \cdot D_{t}^{q} x(t) .
$$

Using (25) in (24),

$$
\begin{aligned}
\dot{V}= & s \cdot D_{t}^{1-q} \cdot D_{t}^{q} s-(b-\widehat{b})\left(D_{t}^{1-q} \cdot D_{t}^{q} \widehat{b}\right) \\
& -(a-\widehat{a})\left(D_{t}^{1-q} \cdot D_{t}^{q} \widehat{a}\right) .
\end{aligned}
$$

Finding the sign of the Lyapunov first derivative using (26) seems difficult and hence we use the modified fractional order Lyapunov method defined by Rajagopal et al. [37] as

$$
\frac{1}{2} D_{t}^{q} x^{2}(t) \leq x(t) \frac{1}{2} D_{t}^{q} x(t), \quad q \in(0,1) .
$$

Using (27), (20), and (18) in (24),

$$
\begin{aligned}
\dot{V} & \leq k s\left[G\left(x_{s}\right)(b-\widehat{b})-F\left(x_{m}\right)(a-\widehat{a})-\eta \operatorname{sgn}(s)\right. \\
& -\rho s]-(b-\hat{b})(\dot{\vec{b}})-(a-\widehat{a})(\dot{\hat{a}}) .
\end{aligned}
$$

Let us define the parameter estimate laws as

$$
\begin{aligned}
& D^{q} \widehat{b}=k s \cdot G\left(x_{s}\right) \\
& D^{q} \widehat{a}=k s \cdot F\left(x_{m}\right) .
\end{aligned}
$$

Using (29) in (28)

$$
\dot{V} \leq-\eta\left|s_{w}\right|-\rho s^{2}
$$

as $\eta$ and $\rho$ are all positive and $\dot{V}$ is negative definite. Using Barbalat's lemma [53], we conclude that $e_{i}(t) \rightarrow 0$ as $t \rightarrow \infty$.

7.1. Adaptive Sliding Mode Synchronization of Identical FOMNE Systems. For synchronization let us define the master system as

$$
\begin{aligned}
& D^{q_{x}} x_{m}=a z_{m}+x_{m} y_{m} \\
& D^{q_{y}} y_{m}=1-x_{m}^{2}-z_{m}^{2}-w_{m}^{2} \\
& D^{q_{z}} z_{m}=-b x_{m}+y_{m} z_{m} \\
& D^{q_{w}} w_{m}=c z_{m}-w_{m}-z_{m} w\left(x_{m}\right)
\end{aligned}
$$

and the slave system with the adaptive controller is defined as

$$
\begin{aligned}
& D^{q_{x}} x_{s}=\widehat{a} z_{s}+x_{s} y_{s}+u_{x} \\
& D^{q_{y}} y_{s}=1-x_{s}^{2}-z_{s}^{2}-w_{s}^{2}+u_{y} \\
& D^{q_{z}} z_{s}=-\widehat{b} x_{s}+y_{s} z_{s}+u_{z} \\
& D^{q_{w}} w_{s}=\widehat{c} z_{s}-w_{s}-z_{s} w\left(x_{s}\right)+u_{w}
\end{aligned}
$$

where $\widehat{a}, \widehat{b}$, and $\widehat{c}$ are the unknown system parameters and $u_{x}$, $u_{y}, u_{z}$, and $u_{w}$ are the adaptive controllers.

The synchronization error dynamics are defined as

$$
\begin{aligned}
& D^{q_{x}} e_{x}=D^{q_{x}} x_{s}-D^{q_{x}} x_{m} \\
& D^{q_{y}} e_{y}=D^{q_{y}} y_{s}-D^{q_{y}} y_{m} \\
& D^{q_{z}} e_{z}=D^{q_{z}} z_{s}-D^{q_{z}} z_{m} \\
& D^{q_{w}} e_{w}=D^{q_{w}} w_{s}-D^{q_{w}} w_{m},
\end{aligned}
$$

where $e_{x}, e_{y}, e_{z}$, and $e_{w}$ are the synchronization errors given by

$$
e_{i}=i_{s}-i_{m}
$$

and $i=x, y, z, w, s$, and $m$ are slave and master systems, respectively.

Using (31) and (32) in (33), we simplify error dynamics as

$$
\begin{aligned}
& D^{q_{x}} e_{x}= \widehat{a} z_{s}+x_{s} y_{s}-a z_{m}-x_{m} y_{m}+u_{x} \\
& D^{q_{y}} e_{y}=-x_{s}^{2}-z_{s}^{2}-w_{s}^{2}+x_{m}^{2}+z_{m}^{2}+w_{m}^{2}+u_{y} \\
& D^{q_{z}} e_{z}=-\widehat{b} x_{s}+y_{s} z_{s}+b x_{m}-y_{m} z_{m}+u_{z} \\
& D^{q_{w}} e_{w}=\widehat{c} z_{s}-w_{s}-z_{s} w\left(x_{s}\right)-c z_{m}+w_{m} \\
& \quad+z_{m} w\left(x_{m}\right)+u_{w} .
\end{aligned}
$$

The parameter estimation errors are defined as

$$
\begin{aligned}
& e_{a}=a-\widehat{a} ; \\
& e_{b}=b-\widehat{b} ; \\
& e_{c}=c-\widehat{c} .
\end{aligned}
$$

The first derivatives of (36) are

$$
\begin{aligned}
& D^{q} e_{a}=-D^{q} \widehat{a} \\
& D^{q} e_{b}=-D^{q} \widehat{b} \\
& D^{q} e_{c}=-D^{q} \widehat{c} .
\end{aligned}
$$

Let us define the integral sliding surface as

$$
s_{i}=e_{i}+k_{i} \int e_{i}(\tau) d \tau
$$


where $i=x, y, z, w$ are the states, $k_{i}>0, s_{i}$ is the sliding surface, and $e_{i}$ is the synchronization errors. The first derivative of the sliding surface (38) can be derived as

$$
D^{q_{i}} s_{i}=D^{q_{i}} e_{i}+k_{i} e_{i} .
$$

Let us define the Lyapunov candidate function as

$$
V=\frac{1}{2}\left[s_{x}^{2}+s_{y}^{2}+s_{z}^{2}+s_{w}^{2}+e_{a}^{2}+e_{b}^{2}+e_{c}^{2}\right] .
$$

The first derivative of the Lyapunov candidate function (40) is

$$
\dot{V}=s_{x} \dot{s}_{x}+s_{y} \dot{s}_{y}+s_{z} \dot{s}_{z}+s_{w} \dot{s}_{w}+e_{a} \dot{e}_{a}+e_{b} \dot{e}_{b}+e_{c} \dot{e}_{c} .
$$

By definition of fractional calculus $[29,30]$,

$$
\dot{x}(t)=D_{t}^{1-q} \cdot D_{t}^{q} x(t) .
$$

Applying (42) in (41)

$$
\begin{aligned}
\dot{V}= & s_{x} D_{t}^{1-q} \cdot D_{t}^{q} s_{x}+s_{y} D_{t}^{1-q} \cdot D_{t}^{q} s_{y}+s_{z} D_{t}^{1-q} \cdot D_{t}^{q} s_{z} \\
& +s_{w} D_{t}^{1-q} \cdot D_{t}^{q} s_{w}+e_{a} D_{t}^{1-q} \cdot D_{t}^{q} e_{a}+e_{b} D_{t}^{1-q} \\
& \cdot D_{t}^{q} e_{b}+e_{c} D_{t}^{1-q} \cdot D_{t}^{q} e_{c} .
\end{aligned}
$$

As observed in (43), analysis of the Lyapunov first derivative is difficult; hence we use the modified fractional order Lyapunov method defined by Rajagopal et al. [37] as

$$
\frac{1}{2} D_{t}^{q} x^{2}(t) \leq x(t) \frac{1}{2} D_{t}^{q} x(t), \quad q \in(0,1) .
$$

Applying (44) in (41),

$$
\begin{aligned}
\dot{V} & \leq s_{x} D^{q_{x}} s_{x}+s_{y} D^{q_{y}} s_{y}+s_{z} D^{q_{z}} s_{z}+s_{w} D^{q_{x}} s_{x} \\
& +e_{a} D^{q} e_{a}+e_{b} D^{q} e_{b}+e_{c} D^{q} e_{c} \\
\dot{V} & \leq s_{x}\left[\widehat{a} z_{s}+x_{s} y_{s}-a z_{m}-x_{m} y_{m}+u_{x}+k_{x} e_{x}\right] \\
& +s_{y}\left[-x_{s}^{2}-z_{s}^{2}-w_{s}^{2}+x_{m}^{2}+z_{m}^{2}+w_{m}^{2}+u_{y}\right. \\
& \left.+k_{y} e_{y}\right]+s_{z}\left[-\widehat{b} x_{s}+y_{s} z_{s}+b x_{m}-y_{m} z_{m}+u_{z}\right. \\
& \left.+k_{z} e_{z}\right]+s_{w}\left[\widehat{c} z_{s}-w_{s}-z_{s} w\left(x_{s}\right)-c z_{m}+w_{m}\right. \\
& \left.+z_{m} w\left(x_{m}\right)+u_{w}+k_{w} e_{w}\right]-e_{a} D^{q} \widehat{a}-e_{b} D^{q} \widehat{b} \\
& +e_{c} D^{q} \widehat{c} .
\end{aligned}
$$

After some mathematical simplifications,

$$
\begin{aligned}
\dot{V} & \leq s_{x}\left[\widehat{a} e_{z}+x_{s} y_{s}-x_{m} y_{m}+u_{x}+k_{x} e_{x}\right]+s_{y}\left[-x_{s}^{2}\right. \\
& \left.-z_{s}^{2}-w_{s}^{2}+x_{m}^{2}+z_{m}^{2}+w_{m}^{2}+u_{y}+k_{y} e_{y}\right] \\
& +s_{z}\left[-\widehat{b} e_{x}+y_{s} z_{s}-y_{m} z_{m}+u_{z}+k_{z} e_{z}\right]+s_{w}\left[\widehat{c} e_{z}\right. \\
& \left.-w_{s}-z_{s} w\left(x_{s}\right)+w_{m}+z_{m} w\left(x_{m}\right)+u_{w}+k_{w} e_{w}\right] \\
& -s_{x} z_{m} e_{a}+s_{z} x_{m} e_{b}-s_{w} z_{m} e_{c}-e_{a} D^{q} \widehat{a}-e_{b} D^{q} \widehat{b} \\
& +e_{c} D^{q} \widehat{c} .
\end{aligned}
$$

Let us define the adaptive controllers as

$$
\begin{aligned}
u_{x}= & -\widehat{a} e_{z}-x_{s} y_{s}+x_{m} y_{m}-k_{x} e_{x}-\eta_{x} \operatorname{sgn}\left(s_{x}\right) \\
& -\rho_{x} s_{x} \\
u_{y}= & x_{s}^{2}+z_{s}^{2}+w_{s}^{2}-x_{m}^{2}-z_{m}^{2}-w_{m}^{2}-k_{y} e_{y} \\
& -\eta_{y} \operatorname{sgn}\left(s_{y}\right)-\rho_{y} s_{y} \\
u_{z}= & \widehat{b} e_{x}-y_{s} z_{s}+y_{m} z_{m}-k_{z} e_{z}-\eta_{z} \operatorname{sgn}\left(s_{z}\right)-\rho_{z} s_{z} \\
u_{w}= & -\widehat{c} e_{z}+w_{s}+z_{s} w\left(x_{s}\right)-w_{m}-z_{m} w\left(x_{m}\right)-k_{w} e_{w} \\
& -\eta_{w} \operatorname{sgn}\left(s_{w}\right)-\rho_{w} s_{w} .
\end{aligned}
$$

And parameter estimation adaptive laws can be defined as

$$
\begin{aligned}
& D^{q} \widehat{a}=-s_{x} z_{m} \\
& D^{q} \widehat{b}=-s_{z} x_{m} \\
& D^{q} \widehat{c}=-s_{w} z_{m} .
\end{aligned}
$$

Using the adaptive controllers (47) and the parameter estimation laws (48) in (46),

$$
\begin{aligned}
\dot{V} \leq & -\eta_{x}\left|s_{x}\right|-\eta_{y}\left|s_{y}\right|-\eta_{z}\left|s_{z}\right|-\eta_{w}\left|s_{w}\right|-\rho_{x} s_{x}^{2} \\
& -\rho_{y} s_{y}^{2}-\rho_{z} s_{z}^{2}-\rho_{w} s_{w}^{2}
\end{aligned}
$$

as $\eta_{x}, \eta_{y}, \eta_{z}$, and $\eta_{w}$ and $\rho_{x}, \rho_{y}, \rho_{z}$, and $\rho_{w}$ are all positive and $\dot{V}$ is negative definite. Using Barbalat's lemma [53], we conclude that $e_{i}(t) \rightarrow 0$ as $t \rightarrow \infty$. For numerical simulations we take the initial conditions as $x_{m}(0)=1, y_{m}(0)=-1$, $z_{m}(0)=-1, w_{m}(0)=1, x_{s}(0)=-2, y_{s}(0)=3, z_{s}(0)=-2$, $w_{s}(0)=4$, and $\widehat{a}=1, \widehat{b}=4$, and $\widehat{c}=13$. Figure 8 shows the time history of the synchronization errors. Figure 9 shows the adapted parameters.

\section{Genetically Optimized Fractional Order PID (FOPID) Controller for the Synchronization of FOMNE Systems}

In this section we investigate the synchronization of FOMNE systems using genetically optimized (GA) FOPID controllers $\left(u_{x}, u_{y}, u_{z}, u_{w}\right)[38,44,45]$ implemented in feedback loops given by

$$
u_{i}=K_{P} e_{i}+K_{I} \int_{0}^{t} e_{i} d \tau^{\beta}+K_{D} \frac{d^{\delta} e_{i}}{d t^{\delta}}, \quad i=x, y ; z ; w,
$$

where $u_{i}$ is the fractional order PID action control for $i=$ $x, y, z, w$ and $\delta, \beta$ are the fractional order differential and integral operators, $e_{i}$ is the error signal, and $K_{P}, K_{I}$, and $K_{D}$ are the proportional, integral, and the derivative gains to synchronize the fractional order model of the memristor no equilibrium system (1). We use genetic algorithm $[54,55]$ to optimize the controller gains such that the error $e_{i}$ is minimized. Matlab tools are used for numerical simulations with the following constraints defined. 


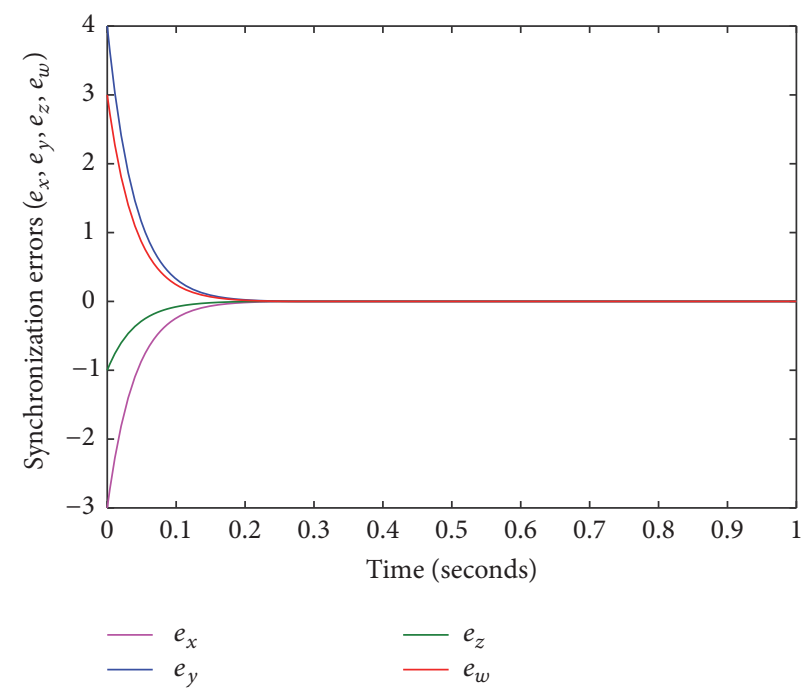

FIGURE 8: Time history of synchronization errors $\left(e_{x}, e_{y}, e_{z}, e_{w}\right)$.

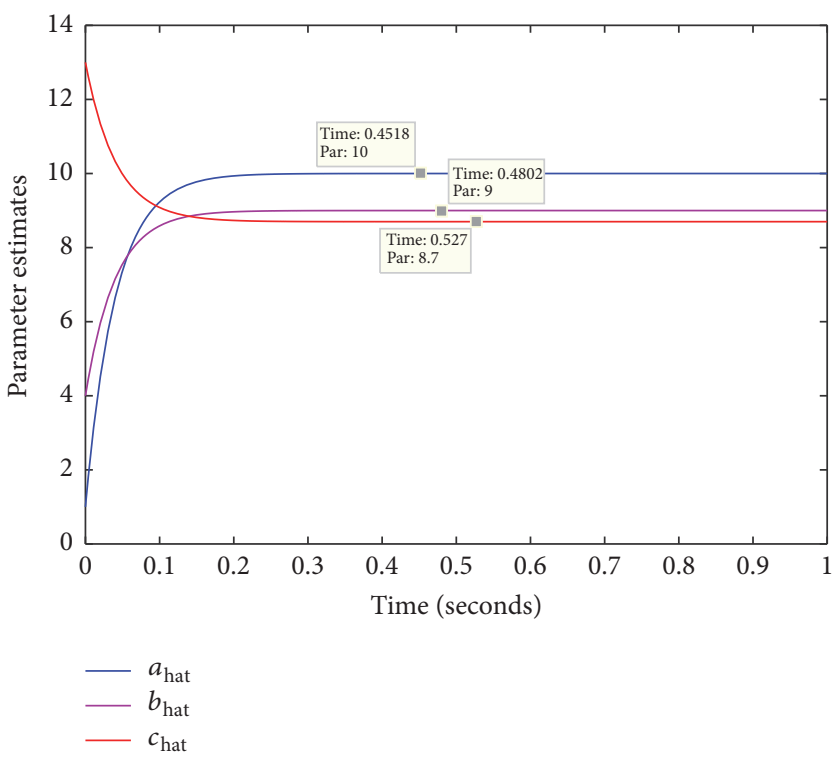

Figure 9: Estimated Parameters $(a, b, c)$.

Variable bounds matrix of the proportional, integral, and the derivative gains $=[-0.001,0.001]$; population size $=80$, GA. Genetically, bigger population size give better approximation; number of generations $=100$; selection function $=$ stochastic uniform; crossover fraction $=0.8$; mutation function $=$ Gaussian; stopping criteria $=$ error performance criterion; length of the chromosome $=12$ (decimal codage).

Tuning of PID controllers involves the selection of $K_{P}, K_{I}$, and $K_{D}$ gains for better control performance which is defined with reference to the required performance index. There are two important performance indices ISE (Integrated Squared Error) and IAE (Integrated Absolute Error) as given in the following:

$$
\begin{aligned}
\mathrm{ISE} & =\int_{0}^{\infty} e_{i}^{2}(\tau) d \tau, \\
\mathrm{IAE} & =\int_{0}^{\infty}\left|e_{i}(\tau)\right| d \tau .
\end{aligned}
$$

In this paper we use IAE as the objective function and the fitness functions is as given below 


$$
\begin{aligned}
& \text { fitness function }=\frac{1}{\sum \operatorname{abs}\left(e_{i}\right)}, \\
& \qquad e_{i}=e_{x}+e_{y}+e_{z}+e_{w} .
\end{aligned}
$$

We synchronize the master system (31) and the slave system (32) using the GA optimized PID controllers with parameters known (Case 1) and parameters unknown (Case 2).

Case 1 (parameters known). In this section we assume that the parameters of the slave system are known. We take the same master system defined by (31) and the slave system (32) is modified with FOPID controllers as given below.

$$
\begin{aligned}
D^{q_{x}} x_{s} & =a z_{s}+x_{s} y_{s}+u_{x} \\
D^{q_{y}} y_{s} & =1-x_{s}^{2}-z_{s}^{2}-w_{s}^{2}+u_{y} \\
D^{q_{z}} z_{s} & =-b x_{s}+y_{s} z_{s}+u_{z} \\
D^{q_{w}} w_{s} & =c z_{s}-w_{s}-z_{s} W\left(x_{s}\right)+u_{w} .
\end{aligned}
$$

The initial conditions and the parameters are taken as

$$
\begin{aligned}
x_{m}(0) & =1, \\
y_{m}(0) & =-1, \\
z_{m}(0) & =-1, \\
w_{m}(0) & =1, \\
x_{s}(0) & =-2, \\
y_{s}(0) & =3, \\
z_{s}(0) & =-2, \\
w_{s}(0) & =4, \\
a & =10, \\
b & =9, \\
c & =8.7, \\
a_{1} & =4, \\
b_{1} & =0.01 .
\end{aligned}
$$

The synchronization error dynamics are defined as,

$$
\begin{aligned}
& D^{q_{x}} e_{x}=a e_{z}+x_{s} y_{s}-x_{m} y_{m}+u_{x} \\
& D^{q_{y}} e_{x}=x_{m}^{2}-x_{s}^{2}+z_{m}^{2}-z_{s}^{2}+w_{m}^{2}-w_{s}^{2}+u_{y} \\
& D^{q_{z}} e_{x}=-b e_{x}+y_{s} z_{s}-y_{m} z_{m}+u_{z} \\
& D^{q_{w}} e_{x}=c e_{z}-e_{w}-z_{s} W_{s}\left(x_{s}\right)+z_{m} W_{m}\left(x_{m}\right)+u_{w} .
\end{aligned}
$$

Table 1 shows the FOPID gain values of the controllers achieved using genetic algorithm for known parameter values. We get the best solutions tracked over generations for the complete synchronization of the FOMNE systems.
Figure 10 shows the time history of the synchronization errors using genetically optimized fractional order PID controllers; Figure 11 shows the synchronized states and Figure 12 shows the time history of the fractional order PID controllers.

Case 2 (parameters unknown). In this section we assume that the parameters of the slave system are unknown. We take the same master system defined by (31) and the slave system (32) is modified with FOPID controllers as given below.

$$
\begin{aligned}
D^{q_{x}} x_{s} & =\widehat{a} z_{s}+x_{s} y_{s}+u_{x} \\
D^{q_{y}} y_{s} & =1-x_{s}^{2}-z_{s}^{2}-w_{s}^{2}+u_{y} \\
D^{q_{z}} z_{s} & =-\widehat{b} x_{s}+y_{s} z_{s}+u_{z} \\
D^{q_{w}} w_{s} & =\widehat{c} z_{s}-w_{s}-z_{s} W\left(x_{s}\right)+u_{w},
\end{aligned}
$$

where $\hat{a}, \widehat{b}$, and $\widehat{c}$ are the unknown system parameters of the slave system. The initial conditions are taken as

$$
\begin{aligned}
x_{m}(0) & =1, \\
y_{m}(0) & =-1, \\
z_{m}(0) & =-1, \\
w_{m}(0) & =1, \\
x_{s}(0) & =-2, \\
y_{s}(0) & =3, \\
z_{s}(0) & =-2, \\
w_{s}(0) & =4, \\
\widehat{a} & =1, \\
\widehat{b} & =4, \\
\widehat{c} & =13 .
\end{aligned}
$$

The synchronization error dynamics are defined as

$$
\begin{aligned}
D^{q_{x}} e_{x}=\hat{a} z_{s}+x_{s} y_{s}-a z_{m}-x_{m} y_{m}+u_{x} \\
D^{q_{y}} e_{y}=-x_{s}^{2}-z_{s}^{2}-w_{s}^{2}+x_{m}^{2}+z_{m}^{2}+w_{m}^{2}+u_{y} \\
D^{q_{z}} e_{z}=-\hat{b} x_{s}+y_{s} z_{s}+b x_{m}-y_{m} z_{m}+u_{z} \\
D^{q_{w}} e_{w}=\widehat{c} z_{s}-w_{s}-z_{s} w\left(x_{s}\right)-c z_{m}+w_{m} \\
\quad+z_{m} w\left(x_{m}\right)+u_{w}
\end{aligned}
$$

and the parameter update laws are defined as

$$
\begin{aligned}
& D^{q} \widehat{a}=-e_{x} z_{m} \\
& D^{q} \widehat{b}=-e_{z} x_{m} \\
& D^{q} \widehat{c}=-e_{w} z_{m} .
\end{aligned}
$$

Table 2 shows the FOPID gain values achieved using the genetic algorithm optimization technique for unknown parameters. 
TABLE 1: FOPID controller gain values optimized with GA.

\begin{tabular}{lccc}
\hline FOPID controller & $K_{P}$ & $K_{I}$ & $K_{D}$ \\
\hline$u_{x}$ & -0.0271 & -0.0095 & 0.0015 \\
$u_{y}$ & -0.0469 & -0.0809 & -0.0029 \\
$u_{z}$ & -0.0072 & 0.0011 & 0.0146 \\
$u_{w}$ & -0.0199 & -0.0063 & 0.0124 \\
\hline
\end{tabular}

TABLE 2: FOPID controller gain values optimized with GA.

\begin{tabular}{lccc}
\hline FOPID controller & $K_{P}$ & $K_{I}$ & $K_{D}$ \\
\hline$u_{x}$ & -0.0826 & -0.1885 & -0.0836 \\
$u_{y}$ & -0.2150 & -0.1586 & 0.0027 \\
$u_{z}$ & 0.1637 & -0.1444 & 0.0888 \\
$u_{w}$ & -0.2177 & -0.0121 & 0.0304 \\
\hline
\end{tabular}

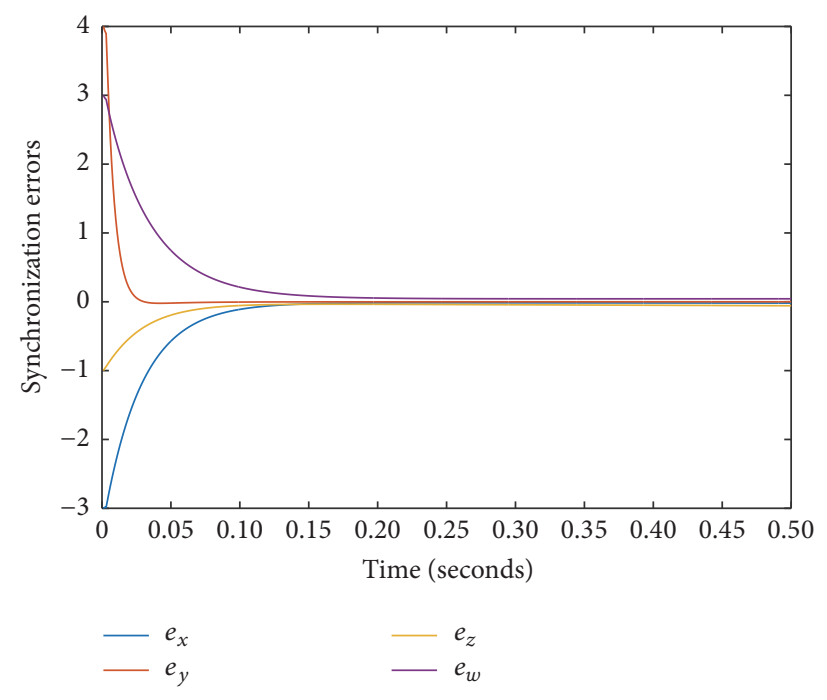

FIgURE 10: Time history of synchronization errors $\left(e_{x}, e_{y}, e_{z}, e_{w}\right)$.

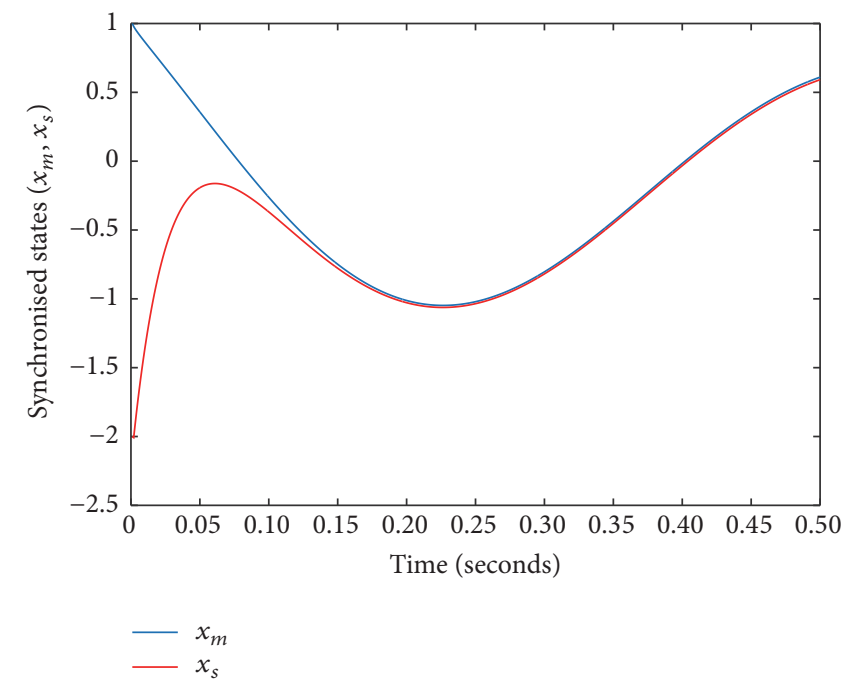

FIgURE 11: State synchronization $\left(x_{m}, x_{s}\right)$. 


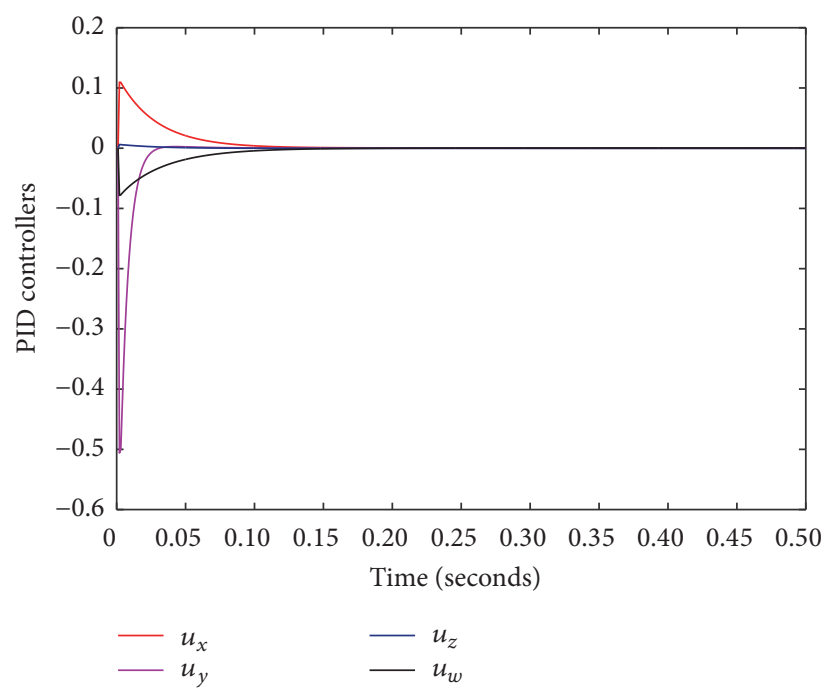

FIgURE 12: Time history of PID controllers $u_{x}, u_{y}, u_{z}$, and $u_{w}$.

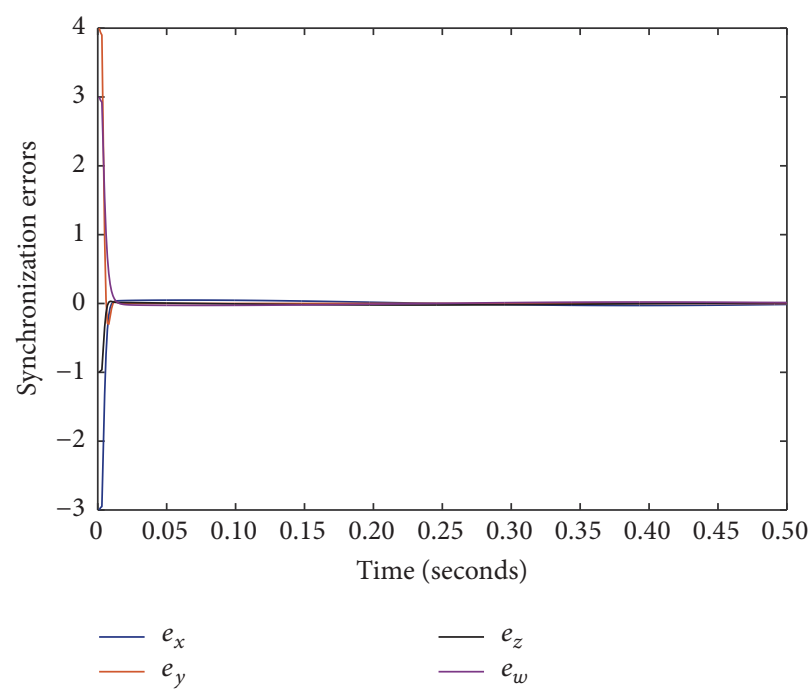

FIGURE 13: Time history of synchronization errors $\left(e_{x}, e_{y}, e_{z}, e_{w}\right)$.

Figure 13 shows the time history of the synchronization errors using genetically optimized fractional order PID controllers, Figure 14 shows the synchronized states, and Figure 15 shows the time history of fractional order PID controllers. Figure 16 shows the time history of the parameter estimates.

As can be seen from Figures 8, 10, and 13, adaptive GA optimized PID control converges much faster $(t=40 \mathrm{mS})$ than adaptive sliding mode control $(t=190 \mathrm{mS})$. Figure 17 shows the comparison of synchronization errors using adaptive sliding mode control (ASMC) and GA optimized PID control (GAPID).

\section{FPGA Implementation of the FOMNE System}

In this section we discuss the implementation of the novel hyperchaotic system in FPGA using the Xilinx (Vivado) system generator toolbox in Simulink. Firstly we configure the available built-in blocks of the system generator toolbox. The Add/Sub blocks are configured with zero latency and $32 / 16$-bit fixed point settings. The output of the block is configured to rounded quantization in order to reduce the bit latency. For the multiplier block a latency of 3 is configured and the other settings are the same as in Add/Sub block. Next we will have to design the fractional order integrator which is not a readily available block in the system generator. Hence we implement the integrators using the mathematical relation discussed in (11) and (12) and the value of $h$ is taken as 0.001 and the initial conditions are fed into the forward register. Figure 18 shows the Xilinx schematics of the FOMNE system (13) implemented in Kintex 7 (device $=7 \mathrm{k} 160 \mathrm{t}$; package $=$ fbg484 S) and Figure 19 shows the Xilinx Kintex 7 schematics of the fractional order integrator. Figure 20(a) shows the power consumed by the FOMNE system and Figure 20(b) shows the power consumption for variation in fractional 


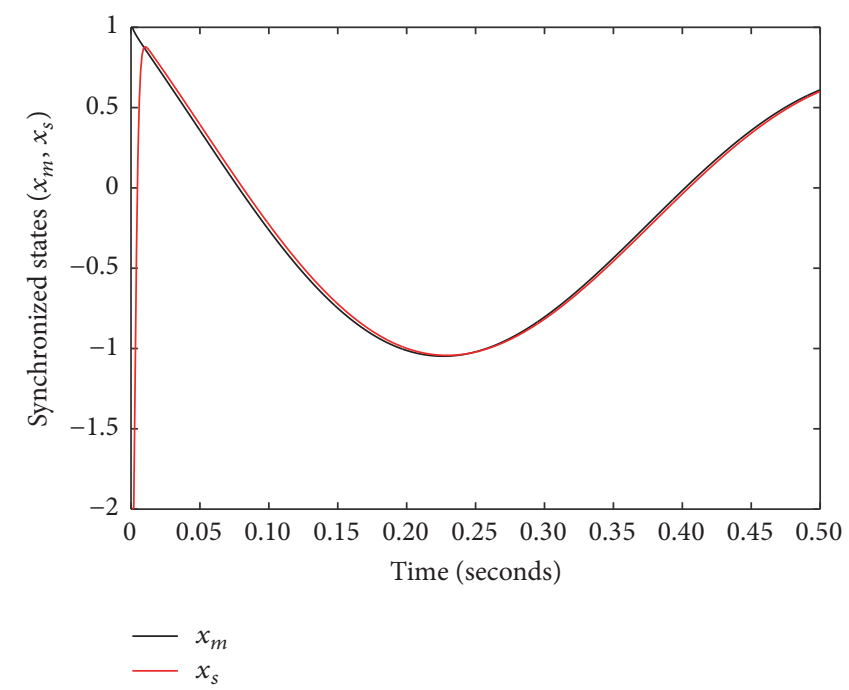

FIGURE 14: State synchronization $\left(x_{m}, x_{s}\right)$.

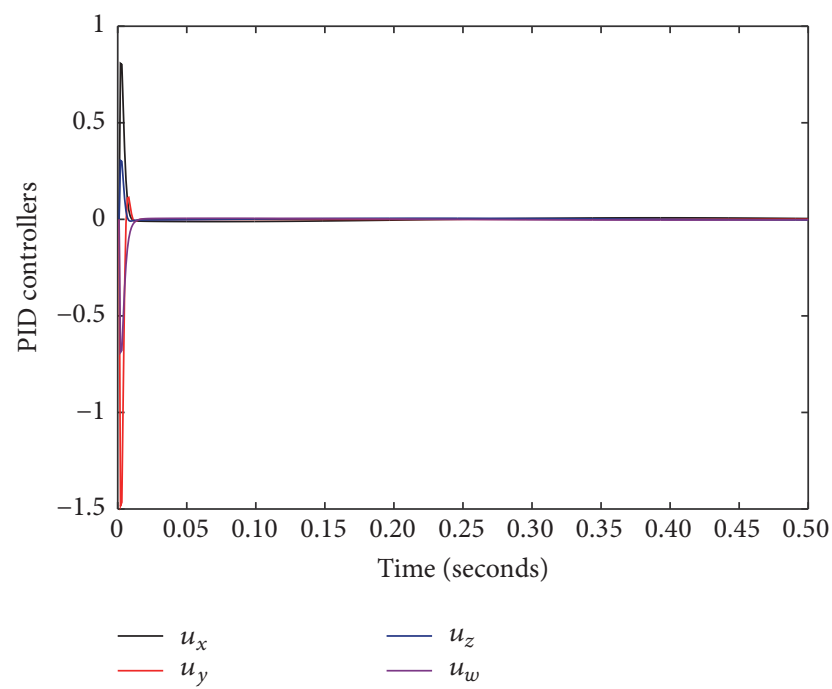

FIGURE 15: Time history of PID controllers $u_{x}, u_{y}, u_{z}$, and $u_{w}$.

orders. As it can be seen from Figure 20(b), the FOMNE system consumes maximum power when its fractional order $q=0.99$ compared to the integer order 1 thus confirming that the fractional order memristor system exhibits largest Lyapunov exponent compared to the integer order. Figure 21 shows the 2D state portraits of the FOMNE system using Xilinx system generator.

\section{Conclusion}

In this paper we have announced a novel $4 \mathrm{D}$ no equilibrium memristor chaotic system. The dynamic properties of the proposed system are investigated to prove the chaotic behavior of the system. The fractional order model of the $4 \mathrm{D}$ no equilibrium memristor chaotic system is derived

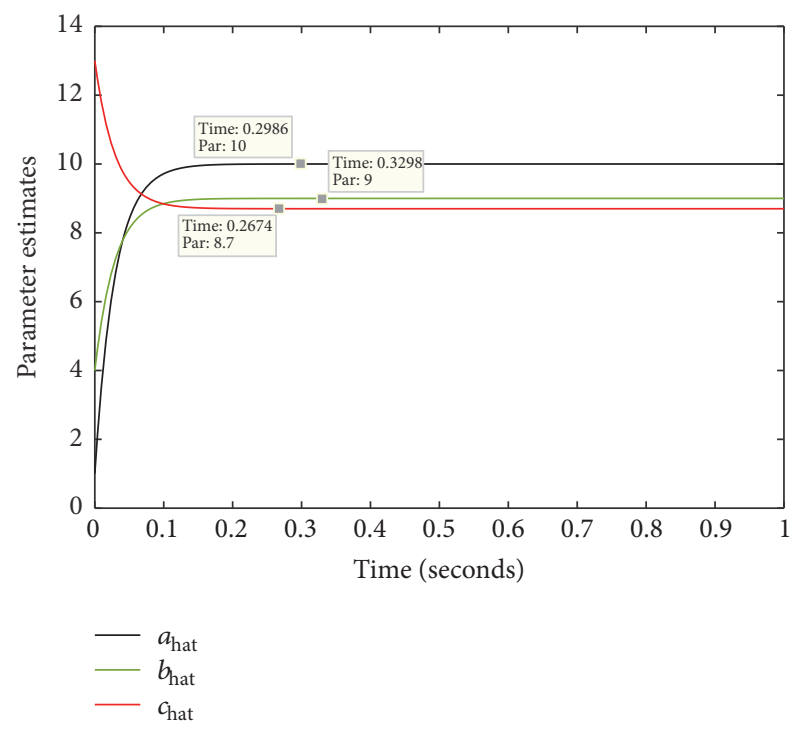

FIGURE 16: Time history of parameter estimates.

from its integer model. Fractional order bifurcation property of the FOMNE system is investigated and it is seen that largest Lyapunov exponent of the system exists when the fractional order is close to 1 . The identical FOMNE systems are synchronized using adaptive sliding mode controllers and genetically optimized PID controllers. Numerical simulations are done to validate the theoretical results. Finally the proposed FOMNE system is implemented in FPGA to show that the system is hardware realizable.

\section{Competing Interests}

The authors declare that there is no conflict of interests regarding the publication of this paper. 


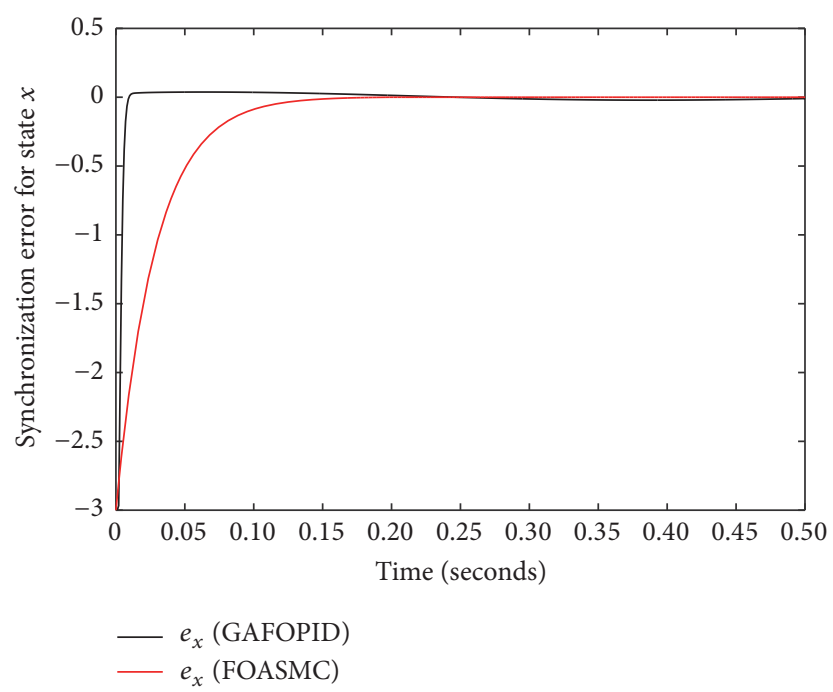

FIGURE 17: Comparison of synchronization errors (ASMC and GAFOPID).

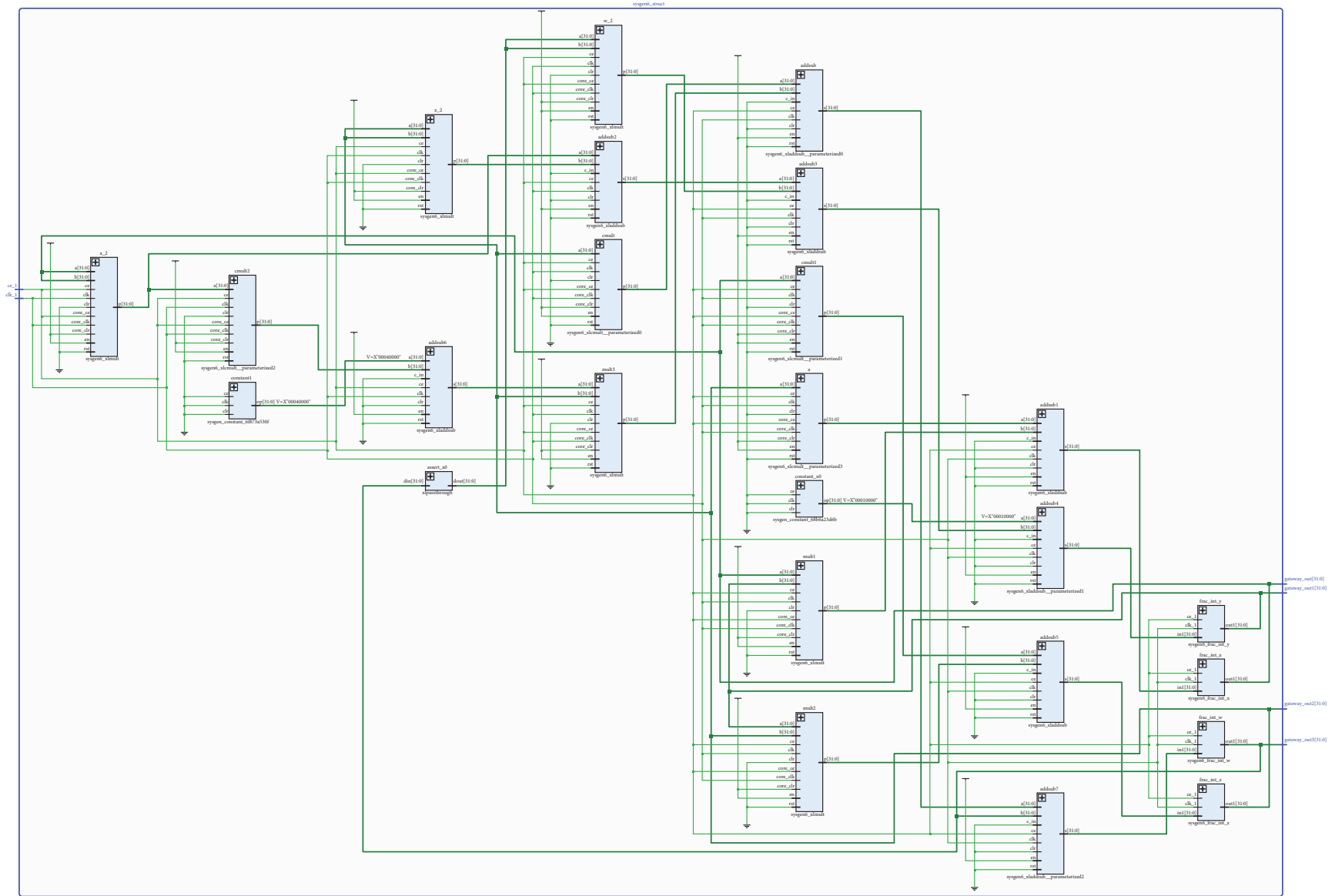

FIgURE 18: Xilinx schematics of the FOMNE system implemented in Kintex 7 (device = 7k160t; package = fbg484 S). 


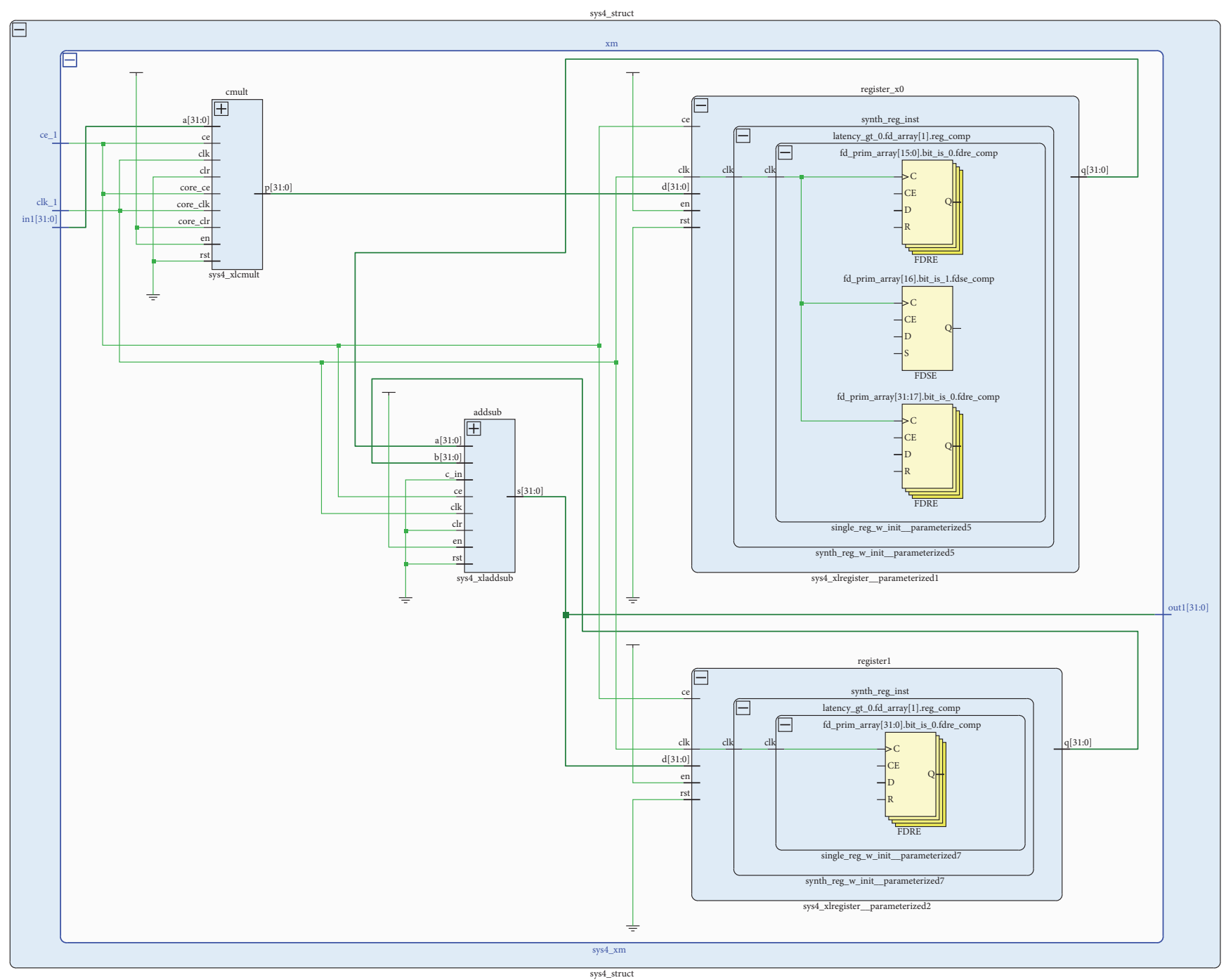

Figure 19: Xilinx schematics of the Fractional order integrator implemented in Kintex 7 (device = 7k160t; package = fbg484 S).

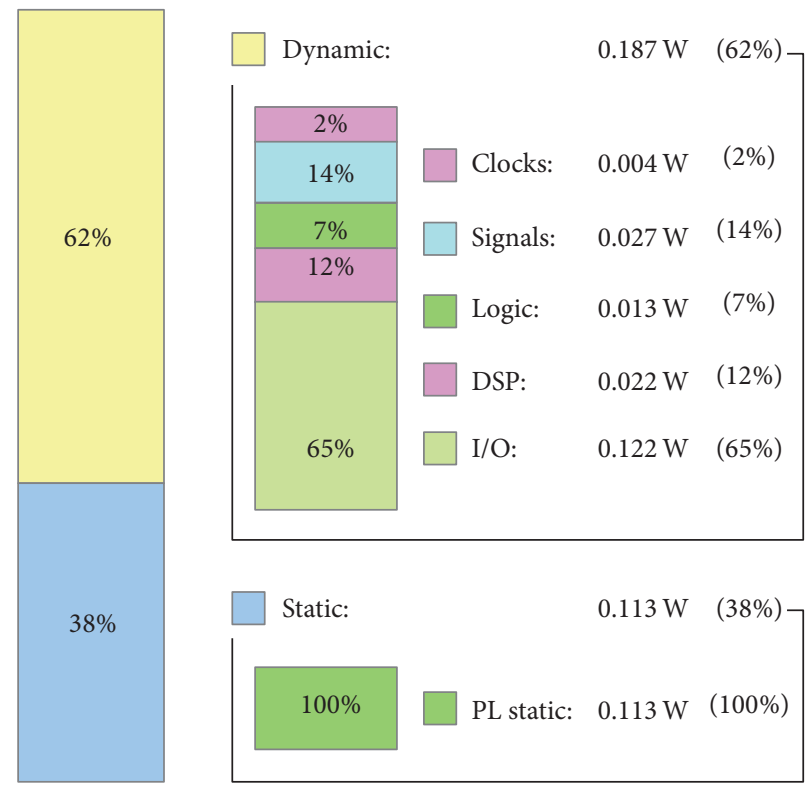

(a)

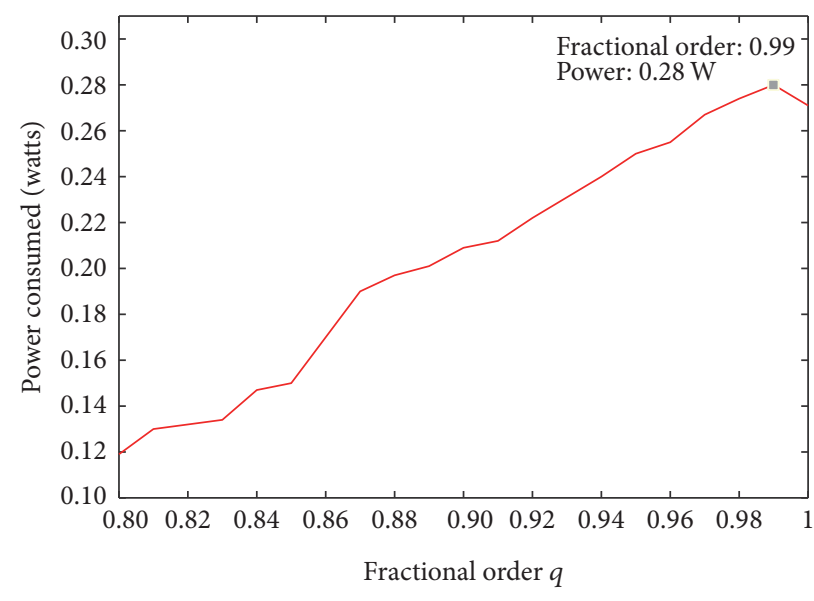

(b)

Figure 20: (a) Power utilized and (b) power utilization versus fractional order of FOMNE system. 

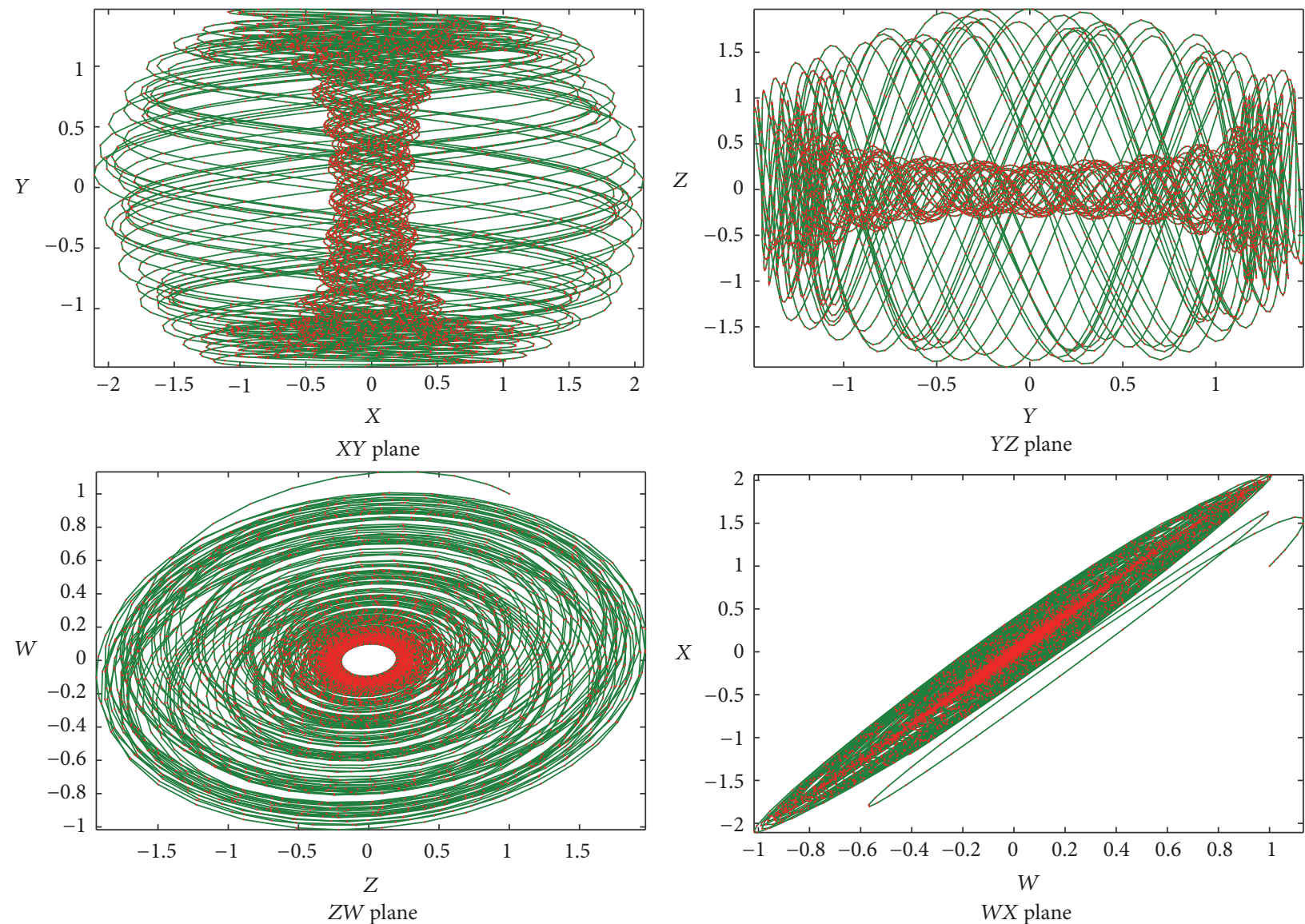

FIGURE 21: 2D state portraits of the FOMENS system implemented using Xilinx system generator (XYZ plane; $Y Z W$ plane).

\section{References}

[1] T. Geisel, “Chaos, randomness and dimension," Nature, vol. 298, no. 5872, pp. 322-323, 1982.

[2] O. E. Rössler, "An equation for hyperchaos," Physics Letters A, vol. 71, no. 2-3, pp. 155-157, 1979.

[3] E. N. Lorenz, "Deterministic nonperiodic flow," Journal of the Atmospheric Sciences, vol. 20, pp. 130-141, 1963.

[4] G. Chen and T. Ueta, "Yet another chaotic attractor," International Journal of Bifurcation and Chaos, vol. 9, no. 7, pp. 14651466, 1999.

[5] C. Liu, T. Liu, L. Liu, and K. Liu, "A new chaotic attractor," Chaos, Solitons and Fractals, vol. 22, no. 5, pp. 1031-1038, 2004.

[6] V. Sundarapandian and I. Pehlivan, "Analysis, control, synchronization, and circuit design of a novel chaotic system," Mathematical and Computer Modelling, vol. 55, no. 7-8, pp. 1904-1915, 2012.

[7] V. Sundarapandian, "Analysis and anti-Synchronization of a novel chaotic system via active and adaptive controllers," Journal of Engineering Science and Technology Review, vol. 6, no. 4, pp. 45-52, 2013.

[8] V.-T. Pham, C. Volos, S. Jafari, Z. Wei, and X. Wang, "Constructing a novel no-equilibrium chaotic system," International Journal of Bifurcation and Chaos, vol. 24, no. 5, Article ID 1450073, 2014.
[9] S. Vaidyanathan and C. Volos, "Analysis and adaptive control of a novel 3-D conservative no-equilibrium chaotic system," Archives of Control Sciences, vol. 25, no. 3, pp. 333-353, 2015.

[10] L. O. Chua, "Memristor-the missing circuit element," IEEE Transactions on Circuit Theory, vol. 18, pp. 507-519, 1971.

[11] L. O. Chua and S. M. Kang, "Memristive devices and systems," Proceedings of the IEEE, vol. 64, no. 2, pp. 209-223, 1976.

[12] Z. Biolek, D. Biolek, and V. Biolková, "SPICE model of memristor with nonlinear dopant drift," Radioengineering, vol. 18, no. 2, pp. 210-214, 2009.

[13] R. E. Pino, J. W. Bohl, N. McDonald et al., "Compact method for modeling and simulation of memristor devices: ion conductor chalcogenide-based memristor devices," in Proceedings of the IEEE/ACM International Symposium on Nanoscale Architectures (NANOARCH '10), pp. 1-4, IEEE, Anaheim, Calif, USA, June 2010.

[14] Á. Rak and G. Cserey, "Macromodeling of the memristor in SPICE," IEEE Transactions on Computer-Aided Design of Integrated Circuits and Systems, vol. 29, no. 4, pp. 632-636, 2010.

[15] A. Ishaq Ahamed and M. Lakshmanan, "Nonsmooth bifurcations, transient hyperchaos and hyperchaotic beats in a memristive Murali-Lakshmanan-Chua circuit," International Journal of Bifurcation and Chaos, vol. 23, no. 6, Article ID 1350098, 2013.

[16] S. Wang, X. Wang, Y. Zhou, and B. Han, "A memristor-based hyperchaotic complex Lü system and its adaptive complex 
generalized synchronization," Entropy, vol. 18, no. 2, article 58, 2016.

[17] B. A. Idowu, U. E. Vincent, and A. N. Njah, "Synchronization of chaos in non-identical parametrically excited systems," Chaos, Solitons and Fractals, vol. 39, no. 5, pp. 2322-2331, 2009.

[18] S. Vaidyanathan and K. Rajagopal, "Hybrid synchronization of hyperchaotic Wang-Chen and hyperchaotic lorenz systems by active non-linear control," International Journal of Systems Signal Control and Engineering Application, vol. 4, no. 3, pp. 5561, 2011.

[19] V. Sundarapandian and R. Karthikeyan, "Anti-synchronization of Lü and Pan chaotic systems by adaptive nonlinear control," European Journal of Scientific Research, vol. 64, no. 1, pp. 94106, 2011.

[20] V. Sundarapandian and R. Karthikeyan, "Adaptive antisynchronization of Uncertain Tigan and Li Systems," Journal of Engineering and Applied Sciences, vol. 7, no. 1, pp. 45-52, 2012.

[21] S. S. Majidabad and H. T. Shandiz, "Discrete-time terminal sliding mode control of chaotic Lorenz system," Journal of Control and Systems Engineering, vol. 1, no. 1, pp. 1-8, 2013.

[22] A. N. Njah, "Tracking control and synchronization of the new hyperchaotic Liu system via backstepping techniques," Nonlinear Dynamics, vol. 61, no. 1-2, pp. 1-9, 2010.

[23] O. S. Onma, O. I. Olusola, and A. N. Njah, "Control and synchronization of chaotic and hyperchaotic lorenz systems via extended backstepping techniques," Journal of Nonlinear Dynamics, vol. 2014, Article ID 861727, 15 pages, 2014.

[24] B. Wang, Y. Li, and D. L. Zhu, "Simplified sliding mode of a novel class of four-dimensional fractional-order chaos," International Journal of Control and Automation, vol. 8, no. 8, pp. 425-438, 2015.

[25] C. Yin, S. Dadras, S.-M. Zhong, and Y. Chen, "Control of a novel class of fractional-order chaotic systems via adaptive sliding mode control approach," Applied Mathematical Modelling, vol. 37, no. 4, pp. 2469-2483, 2013.

[26] H. Liu and J. Yang, "Sliding-mode synchronization control for uncertain fractional-order chaotic systems with time delay," Entropy, vol. 17, no. 6, pp. 4202-4214, 2015.

[27] S. Wang, Y. Yu, and M. Diao, "Hybrid projective synchronization of chaotic fractional order systems with different dimensions," Physica A: Statistical Mechanics and its Applications, vol. 389, no. 21, pp. 4981-4988, 2010.

[28] R. Herrmann, Fractional calculus, World Scientific Publishing Co., Second edition, 2014.

[29] D. Baleanu, K. Diethelm, E. Scalas, and J. J. Trujillo, Fractional Calculus: Models and Numerical Methods, World Scientific, Singapore, 2014.

[30] Y. Zhou, Basic Theory of Fractional Differential Equations, World Scientific, Singapore, 2014.

[31] K. Diethelm, The Analysis of Fractional Differential Equations, Springer, Berlin, Germany, 2010.

[32] X. Wang and G. Chen, "A chaotic system with only one stable equilibrium," Communications in Nonlinear Science and Numerical Simulation, vol. 17, no. 3, pp. 1264-1272, 2012.

[33] X. Wang and G. Chen, "Constructing a chaotic system with any number of equilibria," Nonlinear Dynamics, vol. 71, no. 3, pp. 429-436, 2013.

[34] Z. Wang, S. Cang, E. O. Ochola, and Y. Sun, "A hyperchaotic system without equilibrium," Nonlinear Dynamics, vol. 69, no. 1-2, pp. 531-537, 2012.
[35] G. A. Leonov and N. V. Kuznetsov, "Hidden attractors in dynamical systems: from hidden oscillations in hilbert-kolmogorov, Aizerman, and Kalman problems to hidden chaotic attractor in chua circuits," International Journal of Bifurcation and Chaos, vol. 23, no. 1, Article ID 1330002, 69 pages, 2013.

[36] Z. Wang, X. Huang, and H. Shen, "Control of an uncertain fractional order economic system via adaptive sliding mode," Neurocomputing, vol. 83, pp. 83-88, 2012.

[37] K. Rajagopal, S. Vaidyanathan, A. Karthikeyan, and P. Duraisamy, "Dynamic analysis and chaos suppression in a fractional order brushless DC motor," Electrical Engineering, 2016.

[38] T.-Z. Li, Y. Wang, and M.-K. Luo, "Control of fractional chaotic and hyperchaotic systems based on a fractional order controller," Chinese Physics B, vol. 23, no. 8, Article ID 080501, 2014.

[39] R. H. Li and W. S. Chen, "Fractional order systems without equilibria," Chinese Physics B, vol. 22, Article ID 040503, 2013.

[40] P. Ivo, Fractional Order Chaotic Systems, Matlab Central File Exchange, Math Works, Natick, Mass, USA, 2010, https://www .mathworks.com/matlabcentral/fileexchange/27336.

[41] G. Leonov, N. Kuznetsov, O. Kuznetsova, S. Seldedzhi, and V. Vagaitsev, "Hidden oscillations indynamical systems," Transactions on Systems and Control, vol. 6, pp. 54-67, 2011.

[42] S. Jafari, J. C. Sprott, and S. M. R. Hashemi Golpayegani, "Elementary quadratic chaotic flows with no equilibria," Physics Letters A, vol. 377, no. 9, pp. 699-702, 2013.

[43] I. Petráš, "Fractional-order feedback control of a DC motor," Journal of Electrical Engineering, vol. 60, no. 3, pp. 117-128, 2009.

[44] M. Zamani, M. Karimi-Ghartemani, N. Sadati, and M. Parniani, "Design of a fractional order PID controller for an AVR using particle swarm optimization," Control Engineering Practice, vol. 17, no. 12, pp. 1380-1387, 2009.

[45] A. Djari, T. Bouden, and A. Boulkroune, "Design of a fractional order PID controller, (FOPID) for a class of fractional order MIMO system," Journal of Automation and Systems Engineering, vol. 8, no. 1, pp. 25-39, 2014.

[46] S. Vaidhyanathan and C. Volos, "Analysis and adaptive control of a novel 3-D conservative no-equilibrium chaotic system," Archives of Control Sciences, vol. 25, no. 3, pp. 333-353, 2015.

[47] Q. Li, H. Zeng, and J. Li, "Hyperchaos in a 4D memristive circuit with infinitely many stable equilibria," Nonlinear Dynamics, vol. 79, no. 4, pp. 2295-2308, 2015.

[48] Q.-H. Hong, Y.-C. Zeng, and Z.-J. Li, “Design and simulation of chaotic circuit for flux-controlled memristor and chargecontrolled memristor," Wuli Xuebao/Acta Physica Sinica, vol. 62, no. 23, Article ID 230502, 2013.

[49] B. Bao, P. Jiang, H. Wu, and F. Hu, "Complex transient dynamics in periodically forced memristive Chua's circuit," Nonlinear Dynamics, vol. 79, no. 4, pp. 2333-2343, 2015.

[50] C. Pezeshki, S. Elgar, and R. C. Krishna, "Bispectral analysis of possessing chaotic motion," Journal of Sound and Vibration, vol. 137, no. 3, pp. 357-368, 1990.

[51] M. E. Fouda and A. G. Radwan, "On the fractional-order memristor model," Journal of Fractional Calculus and Applications, vol. 4, no. 1, pp. 1-7, 2013.

[52] Y.-F. Pu and X. Yuan, "Fracmemristor: fractional-order memristor," IEEE Access, vol. 4, pp. 1872-1888, 2016.

[53] H. K. Khalil, Nonlinear Systems, Prentice Hall, New York, NY, USA, 2002. 
[54] L. Guessas and K. Benmahammed, "Adaptive backstepping and pid optimized by genetic algorithm in control of Chaotic," International Journal of Innovative Computing, Information and Control, vol. 7, no. 9, pp. 5299-5312, 2011.

[55] D. E. Goldberg, Genetic Algorithms in Search, Optimization \& Machine Learning, Addison-Wesley, Boston, Mass, USA, 1989. 


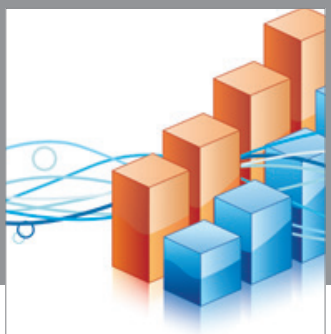

Advances in

Operations Research

vatem alat4

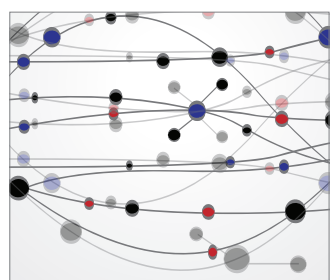

\section{The Scientific} World Journal

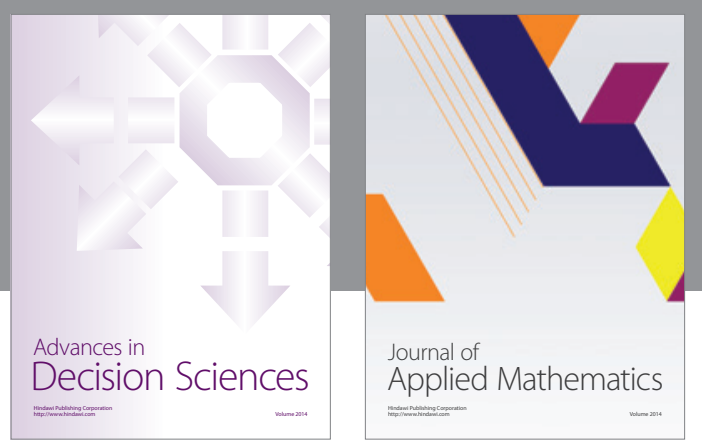

Algebra

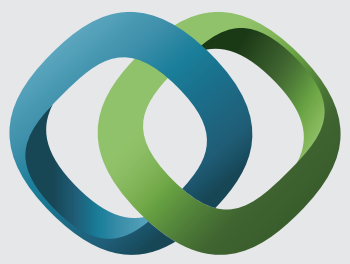

\section{Hindawi}

Submit your manuscripts at

https://www.hindawi.com
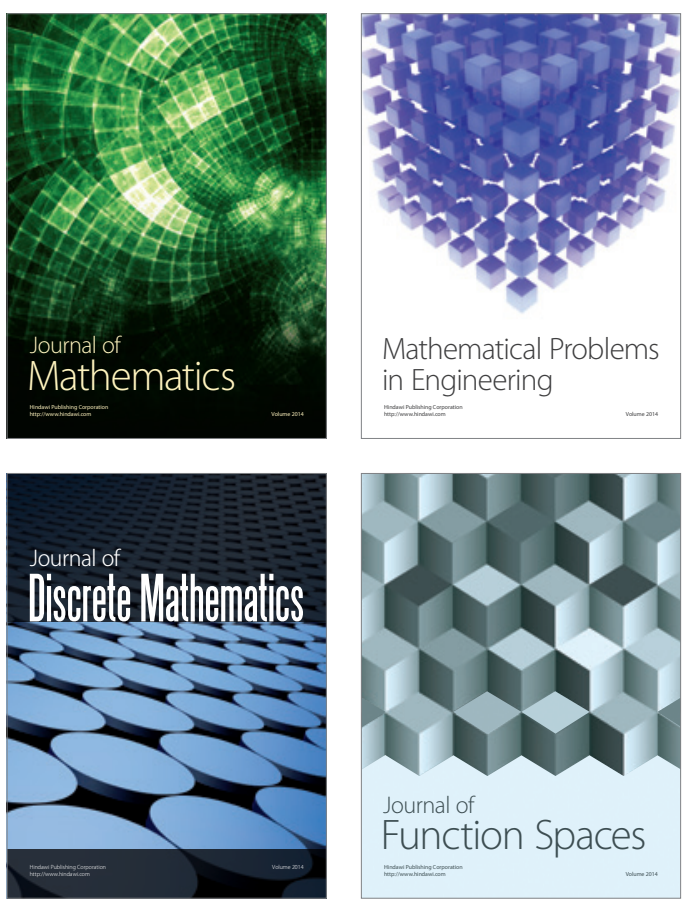

Mathematical Problems in Engineering
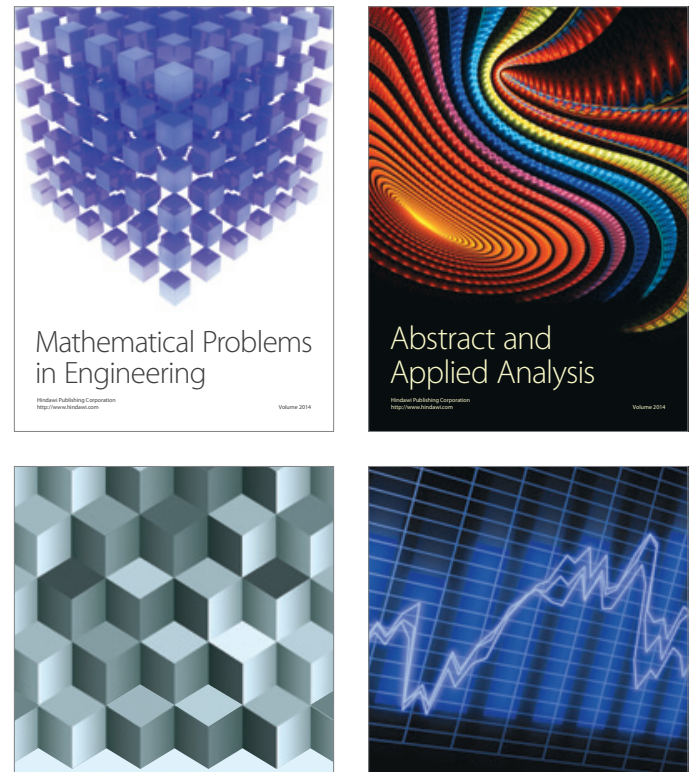

Journal of

Function Spaces

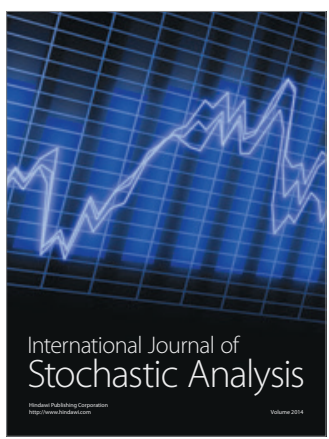

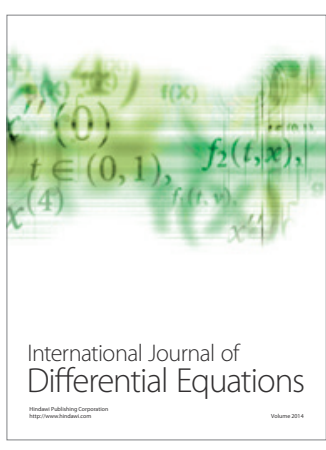
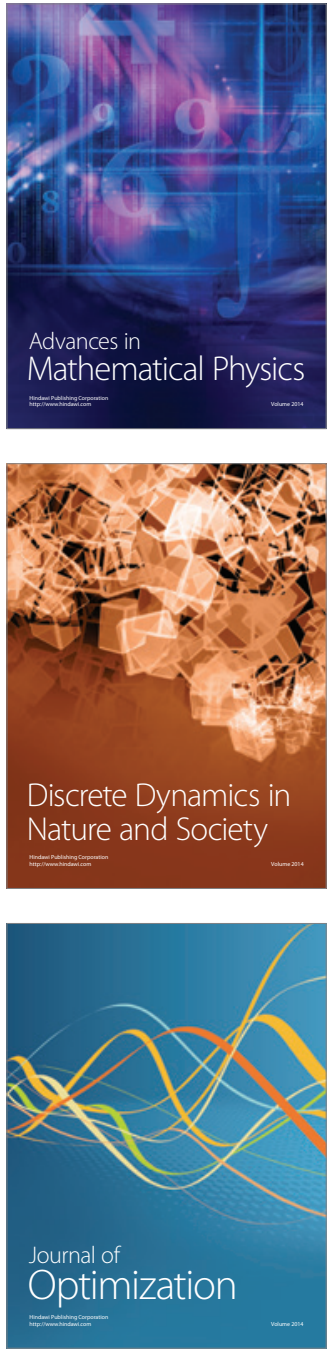